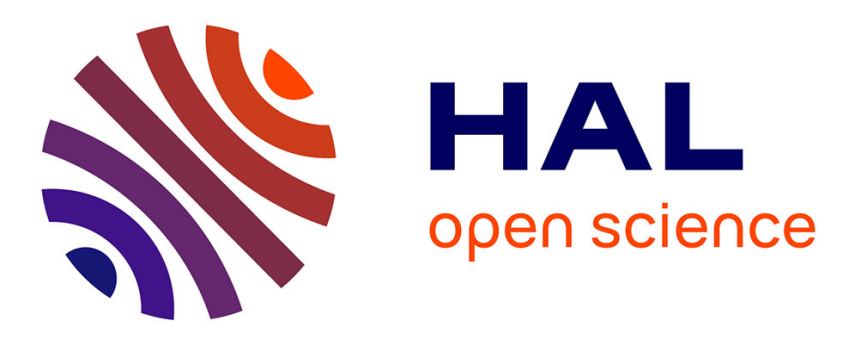

\title{
Modeling of the interaction between bone tissue and resorbable biomaterial as linear elastic materials with voids
}

Ugo Andreaus, Ivan Giorgio, Angela Madeo

\section{- To cite this version:}

Ugo Andreaus, Ivan Giorgio, Angela Madeo. Modeling of the interaction between bone tissue and resorbable biomaterial as linear elastic materials with voids. Zeitschrift für Angewandte Mathematik und Physik, 2015, 66 (1), pp.209-237. 10.1007/s00033-014-0403-z . hal-00944570

\author{
HAL Id: hal-00944570 \\ https://hal.science/hal-00944570
}

Submitted on 10 Feb 2014

HAL is a multi-disciplinary open access archive for the deposit and dissemination of scientific research documents, whether they are published or not. The documents may come from teaching and research institutions in France or abroad, or from public or private research centers.
L'archive ouverte pluridisciplinaire HAL, est destinée au dépôt et à la diffusion de documents scientifiques de niveau recherche, publiés ou non, émanant des établissements d'enseignement et de recherche français ou étrangers, des laboratoires publics ou privés. 


\title{
Modeling of the interaction between bone tissue and re- sorbable biomaterial as linear elastic materials with voids
}

\author{
Ugo Andreaus, Ivan Giorgio and Angela Madeo
}

\begin{abstract}
In this paper a continuum mixture model with evolving mass densities and porosity is proposed to describe the process of bone remodeling in presence of bio-resorbable materials as driven by externally applied loads. From a mechanical point of view, both bone tissue and biomaterial are modeled as linear-elastic media with voids in the sense of [19]. In the proposed continuum model the change of volume fraction related to the void volume is directly accounted for by considering porosity as an independent kinematical field. The bio-mechanical coupling is ensured by the introduction of a suitable stimulus which allows for discriminating between resorption (of both bone and biomaterial) and synthesis (of the sole natural bone) depending on the level of externally applied loads. The presence of a 'lazy zone' associated with intermediate deformation levels is also considered in which neither resorption nor synthesis occur. Some numerical solutions of the integro-differential equations associated with the proposed model are provided for the twodimensional case. Ranges of values of the parameters for which different percentages of biomaterial substitution occur are proposed, namely parameters characterizing initial and maximum values of mass densities of bone tissue and of the bioresorbable material.
\end{abstract}

Keywords. Mechanical-biological coupling, mechanical loading, growth/resorption processes, continuum mixture model, poroelasticity.

\section{Introduction}

Bone in humans and other mammals is generally classified into two types: i) cortical bone, also known as compact bone and ii) trabecular bone, also known as cancellous or spongy bone. These two types of bones are distinguished on the basis of porosity and density. Cortical bone is much denser with a porosity ranging between $5 \%$ and $10 \%$. Trabecular bone, on the other hand, is much more porous with porosity ranging from $50 \%$ to $90 \%$ and it has a lower mass density. Bones can be considered as materials containing large (vasculature) and small (lacuno canalicular system) interconnected fluid-filled pores. It is then clear that porosity can actually macroscopically influence the mechanical properties of bone and so, indirectly, also the process of bone remodeling. Indeed, it is well known that bone adapts to externally applied loads depending on its deformation states: if deformation is too severe, then new bone is synthesized, while resorption takes place for low values of deformation. In this paper, we show how porosity can macroscopically affect the process of bone remodeling in presence of artificial biomaterials. Porosity will be in fact shown to be one of the main parameters influencing the mechanical response of the mixture.

It has been also observed that fluid flow can occur through the interconnected pores of bone (see e.g. [55]), but it is not clear still today whether this flow can actually influence the overall process of bone remodeling. Indeed, it is sensible that fluid flow can have some role in the modalities of transmission of information between cells involved in resorption and synthesis of bone and also in the process of vascularization. Nevertheless, it is not evident at present if fluid flow can also directly 
influence the overall deformation of bone so affecting the final process of remodeling in a quantitative way. In this paper we explicitly consider porosity as an additional kinematical field, but we do not account for the presence of a saturating fluid in the interconnected pores.

Regenerative medicine and tissue engineering are aimed at developing therapeutic approaches that can induce regeneration of organs or tissues affected by trauma or degenerative diseases. In recent years, the efforts of numerous researchers and biotechnological industries have led to the development of new materials and techniques employed for bone reconstruction. This effort is justified by the great benefit that could be achieved from alternative sources for the reconstruction of bone tissue defects and, more specifically, from engineered constructs that can be easily integrated into natural tissues. Treatment and regeneration of damaged tissues or organs is highly problematic due to the limited number of patient's tissues that can be used for transplants. Consequently, there is ongoing interest in developing new bio-materials that can be used as temporary artificial matrices (scaffolds) for tissue regeneration [99]. In the design of these scaffolds, proper models should be considered to describe possible damage occurring in the bone or in the bio-material, see e.g. [24, 21, 32, 88, 22].

Ideally, a scaffold for bone replacement must be porous, bio-active, biodegradable and possess adequate mechanical properties analogous to those of the biological site [98]. To obtain a bone-engineered tissue, the scaffold should hence meet a number of essential requirements, primarily interconnected pores of an adequate size to allow chemotaxis, cell proliferation and differentiation. The scaffold should preferably be biodegradable since its long-term performances in vivo mainly depend on the rate of degradation of the scaffold itself compared to the rate of simultaneous new bone regeneration. However, the right equilibrium between the rate of in vivo biomaterial resorption and natural tissue regeneration is not easy to attain [99]. A high porosity and an adequate pore size are necessary to facilitate cell seeding and diffusion throughout the whole structure of bone. Porosity is also essential for the prompt vascularization of sites treated with artificial biomaterials. Moreover, high porosity and pore interconnectivity are key requisites to increase the specific surface area available for cell attachment and tissue ingrowth, so facilitating the uniform distribution of cells and the adequate transport of nutrients and cellular waste products. Degradation properties are of crucial importance in bio-material selection and design in tissue engineering. The bio-material should not only stimulate and support tissue growth, but it may also be resorbed with the same rate at which new tissue forms, and importantly, it has to possess the additional ability to withstand the loading conditions experienced in situ. The mechanical support is continuously needed as the material degrades, until the new tissue can take up the load [9].

In the model proposed in this paper, we account for the description of all these important biomaterial characteristics, namely porosity, resorption rate, mechanical resistance. We also propose some numerical values for the associated parameters which allow for the description of the desirable situation in which almost complete biomaterial resorption takes place together with simultaneous replacement with new-formed bone. Nevertheless, an experimental setup coupled with the theoretical one presented in this paper is necessary to provide suitable insights towards applications. Experimental tests (in vivo and in vitro) would be needed to estimate realistic values of the parameters associated with the theoretical model presented here. Such experimental tests should be performed by taking into account the presence of the artificial biomaterial and extending the methods presented e.g. in [16]

Natural bone is a complex composite, mainly constituted of biological apatite and molecules of collagen. In this system, collagen serves as the matrix for cell growth and tissue repair, while apatite serves as the inorganic phase to improve mechanical strength and regeneration of bone. In recent years, fabrication of inorganic-organic composites mimicking the composite nature of real bone has attracted research interest in the field of bone regeneration. Materials used for such bone-mimicking composites are usually inorganic ceramics and biopolymers. Biopolymers can be natural (alginate, collagen, hyaluronic acid, gelatin, fibrin, chitosan, etc) or synthetic. As compared to natural biopolymers, synthetic biopolymers can be tailored to control their properties, such as degradation rate and mechanical strength [100]. The use of synthetic polymers for bone regeneration is extremely appealing in the clinical field, since they can be easily fabricated into three-dimensional structures that fit the defect size well, presenting a large surface for cell adhesion and migration and a controlled porosity 
that allows for an adequate diffusion of nutrients and cells. Synthetic biodegradable polymers most frequently used for tissue regeneration include polyesters, polyanhydrides, polyphosphazenes, and some polyurethanes [96, 99]. Synthetic biodegradable polymers based on polyesters, such as Poly Lactic Acid and Poly Glycolic Acid, have been used in many clinical applications. Hydroxyapatite, the main mineral component of bone, is a naturally occurring mineral form of calcium phosphate. Bioceramics based on calcium phosphate, mainly Hydroxyapatite and tricalcium phosphate in the crystal form $\beta$, are hence currently employed for bone substitute applications [97]. Indeed, we want to stress the fact that the two introduced big classes of bio-materials, polymers and ceramics, are known to be degraded with different mechanisms by the organism. While polymers are mainly degraded in the process of hydrolysis [93, 92], organic ceramics are instead mostly processed by the osteoclasts [94, 84]. In practice, both processes (hydrolysis and osteoclastic resorption) always coexist and, depending on the constitution of the graft, one process or the other results to be dominant.

The idea of considering degradation of polymeric bio-materials via hydrolysis is interesting and it would deserve to be exploited also in the framework of the macroscopic model presented in the present paper. Nevertheless, this is not the primary aim of our paper in which we limit to consider resorption by osteoclasts and we keep this possibility open for future investigations. In the view of application, we can say that the biomaterial targeted in the framework of this paper is a $\beta$-TCP (Tri-Calcium Phosphate) with 5\% of HAP (Hydroxyapatite). This type of material can be resorbed by osteoclasts and is such that degradation by hydrolysis can be considered negligible so that our model can be naturally applied.

To the sake of completeness, we also say that there is a recent and fast-growing interest in the use of biodegradable metals for biomedical applications [98] which are important for proper design of prosthetic devices in contact with bone (e.g. fracture fixation plates, surgical screws, and artificial joints $[6,7,56,26])$.

Following the philosophy put forward by Epstein and Maugin [42], DiCarlo and Quiligotti [41], Grillo et al. [54] presented a model of growth and mass transfer in multi-constituent materials in which the variations of mass of the constituents, and the related structural reorganization of the material, were accompanied by inelastic deformations, and determined the rates of these on the basis of thermodynamic arguments.

It is well known and it will be duly explained in the body of this paper, that the level of mechanical deformation of the natural-bone/biomaterial mixture strongly influences the final percentage of replacement of biomaterial with new-formed bone. It is then natural that, in view of subsequent developments, an 'intelligent' bio-resorbable material could be designed aiming to much more effective performances by using the concept developed in $[8,36,37,73,74,52,75,89,90]$ which allow passive control of deformation; analogously a smart graft could be conceived which is capable to transform biochemical energy into mechanical energy in a cellular environment [12]. How it will be better explained in the following, we consider in this paper the possibility of describing the effect on bone remodeling of both sensor cells (measuring deformation at the point in which they are located and emitting a signal proportional to its intensity) and actor cells (collecting the signal and resorbing or synthesizing depending on the entity of deformation). This paper thus generalizes the model presented in $[5,4,3,1]$ in which the single cellular automata concentrates both sensorial and actuatorial functions.

It is worth noting that the phenomena of resorption or synthesis in bones and resorption in biomaterials due to the bio-mechanical evolution are similar to phase transitions studied in $[43,44,45,46$, $47,81]$. Moreover, the remodeling process presents some stability and bifurcation problems, especially when the applied load reaches a certain level in the presence of geometric or material nonlinearities. In a few papers, $[64,65,85,79]$, for cases of similar complexity, these difficulties are treated. It has to be remarked that at least two time scales are of relevance in the considered context: the characteristic time of bone growth and resorption and the characteristic time of varying externally applied loads. Multiple time-scale analyses, see e.g. [61, 62, 63], can be used to dealt with this aspect.

The present paper is inserted in a recent fruitful research activity on the subject of bone remodeling in presence of biomaterial [59, 70, 69, 60, 2]. In particular, Madeo et al. in [70] introduced 
a two-constituent porous continuum as a model describing the growth/resorption processes in bone tissues grafted with bio-resorbable materials as driven by mechanical stimulus, under the assumption of first-order deformation theory. Madeo et al. in [69] presented an improvement of the previous model [70] under the assumption of second gradient deformation theory. Some numerical simulations for rod-like bones subjected to axial external load are presented in [70, 69]. Lekszycki at al. in [60] proposed a continuum poro-elastic mixture model which applies to 3D case and performed numerical investigations in unidimensional samples to study mechanisms observable during remodeling processes. Andreaus et al. [2] employed the above 3D theoretical model, previously developed and used in 1D space $[60,70]$, to investigate more complex geometries: a continuum model of a mixture of bone tissue and bio-resorbable material was in fact applied to $2 \mathrm{D}$ samples. In the present paper, we add the possibility of explicitly accounting for the presence of porosity in the natural-bone/biomaterial mixture and for the presence of what we call a 'lazy zone'. In particular, while in the quoted papers it is assumed that a threshold for the biological stimulus (related to the entity of deformation) exists discriminating between resorption and synthesis, we suppose here that a lazy zone exists (associated with intermediate levels of deformation) in which neither resorption nor synthesis take place.

Clinical observation shows that some critical situations are exhibited when grafted material may be too fast resorbed, thus allowing for residual gaps in newly formed bone. It is therefore not without interest and hence it is the aim of this paper to study in which conditions and to what extent the functional adaptation leads to a substitution of bio-resorbable material with bone tissue. To accomplish this task, the linear theory of elastic materials with voids developed by Cowin and Nunziato [19] is herein employed to describe the mechanical behaviour of a mixture of bone tissue and bioresorbable material, where the change in void volume is induced by deformations (e.g. the results in $[30,34,83,68,95,82,29,71,67,35,25]$ are useful to model complex porous materials). In the model presented here the mixture can be intended as a solid material with small distributed voids which contain nothing of mechanical or energetic significance. The basic premise underlying the theory to which we are inspired is the concept of a material for which the bulk density is written as the product of two fields, the density field of the matrix material and the volume fraction field. This representation of the bulk density of the material introduces an additional degree of kinematic freedom in the theory with respect to what done in $[70,69,60]$. Moreover, the modeling of the interface between the existing bone tissue and the bio-resorbable material used as a scaffold for tissue growth has to be examined with particular attention to determine the final bone properties. Indeed, the initial discontinuity surface could persist at the end of the remodeling process. The results presented in $[31,28,32,38,33,39,40]$ could be referred to as far as interfaces and continuum models for physical properties of interfaces are concerned. Similar approaches using micromorphic models are also available (see e.g. [66, 78, 77, 76, 51]) and could be also be considered in the same optic.

A discrete modeling of lattices alternative to the present continuum model of mixture can be found in $[88,87,86]$.

The perspective of the main phenomena occurring in the reconstructed bones could be outlined as follows. In this article, we assume that the mechanical stimulus that drives the process of remodeling of bone and biomaterial is the energy density of elastic deformation. According credited conjectures, alternatively the stimulus may be formed by the energy density of viscous dissipation. In any case, it is assumed the existence of an area called lazy zone, such that, if the stimulus falls within it, there is a situation of substantial dynamic equilibrium between synthesis and resorption, but if the stimulus exceeds the upper bound of the lazy zone, it activates the synthesis of the bone, whereas if the stimulus falls below the lower bound, resorption of bone and biomaterial is activated. The stimulus is the signal that is sensed by the sensor cells and transmitted to actuator cells, which synthesize and resorb bone, and resorb the biomaterial, depending on the level of stimulus with respect to the lazy zone. In this article it is assumed that the signal is transmitted with infinite speed. In this regard credited conjectures have been put forward about the phenomenon of the spread of a hormone-like chemical messenger that carries the signal at finite speed and activates the undifferentiated precursor cells that are already on site, and which differentiate into osteoblasts or osteoclasts depending on the level of the 
stimulus with respect to the lazy zone. Herein the assumption was made that the precursor cells have already colonized uniformly the space occupied by biomaterial, assuming that the time it takes for this to happen has already expired. More generally, the colonization may be not uniform and incomplete, in dependence of the size of the graft and of the characteristic times of remodeling. As for the proposed model, the assumption was made that the pores are empty and dilate or contract in dependence of external loads. The motion of the body fluid that flows into the interstitial lacuno-canalicular network should also take into account. The ambitious goal could be to optimize the parameters characterizing the biomaterial, namely bulk density, and therefore stiffness and strength, and rate of resorption, in order to obtain the resorption of the biomaterial simultaneously with the synthesis of the bone, in such a way that gaps do not occur, even if only temporarily, and the mixture always has sufficient stiffness and strength, in order to avoid risks of excessive deformation or of local or even global failure.

\section{Methods}

\subsection{Mechanical coupling between displacement and change of matrix volume fraction}

In this paper, a variational principle is used for the continuum theory of materials with voids proposed in [20]. The theory consisted of constitutive equations and balance equations which determined the stresses in flow situations as well as in equilibrium. As in classical theory a reference configuration can be fixed in an arbitrary way and a motion may be written in the form

$$
\boldsymbol{x}=\chi(\mathbf{X}, t),
$$

where $\mathbf{X}$ is the position of a particle of the body in its reference configuration, and $\boldsymbol{x}$ the spatial position occupied in the current configuration at the time $t$ by the particle labeled $\mathbf{X}$. A significant feature of the continuum theory for materials with voids is the interpretation of the apparent density $\rho(\boldsymbol{x}, t)$, in the instantaneous configuration, as a bulk density expressible as the product of the fields $\gamma(\boldsymbol{x}, t)$ and $\varsigma(\boldsymbol{x}, t)$

$$
\rho(\boldsymbol{x}, t)=\gamma(\boldsymbol{x}, t) \varsigma(\boldsymbol{x}, t),
$$

where $\gamma(\boldsymbol{x}, t)$ is the actual density of the solid matrix itself at the position $\boldsymbol{x}$ at time $t$, and $\varsigma(\boldsymbol{x}, t)$ is the ratio of the volume occupied by the solid matrix to the bulk volume at the position $\boldsymbol{x}$ at time $t$. The field $\varsigma(\boldsymbol{x}, t)$ was called the volume distribution function or volume fraction, and it is subjected to the restriction $0<\varsigma \leqslant 1$. The volume fraction is related to the porosity $\phi$ by $\varsigma=1-\phi$. The linear theory of elastic materials with voids deals with small changes from a reference configuration of a porous body [53]. In this configuration, the bulk density $\rho^{*}$, matrix density $\gamma^{*}$, and matrix volume fraction $\varsigma^{*}$ are related by ${ }^{1}$

$$
\rho^{*}(\mathbf{X}, t)=\gamma^{*}(\mathbf{X}) \varsigma^{*}(\mathbf{X}, t)
$$

and the body is assumed to be strain-free. The independent kinematic variables are assumed to be the displacement field $u_{\mathrm{i}}(\mathbf{X}, t)$ from the reference configuration and the change in volume fraction from the reference volume fraction, $\xi(\mathbf{X}, t)$,

$$
\xi(\mathbf{X}, t)=\varsigma(\chi(\mathbf{X}, t), t)-\varsigma^{*}(\mathbf{X}, t) .
$$

The small strain tensor $E_{\mathrm{ij}}(\mathbf{X}, t)$ is determined from the displacement field according to

$$
E_{\mathrm{ij}}=\frac{1}{2}\left(u_{\mathrm{i}, \mathrm{j}}+u_{\mathrm{j}, \mathrm{i}}\right) \text {. }
$$

Assuming that the material possesses a center of symmetry and is isotropic, the stored energy density $\mathscr{E}$ associated with strain and void volume distortion from the reference configuration is a positive definite quadratic form [19]:

$$
\mathscr{E}=\frac{1}{2} \lambda E_{\mathrm{ii}} E_{\mathrm{jj}}+\mu E_{\mathrm{ij}} E_{\mathrm{ij}}+\frac{1}{2} K_{1} \xi^{2}+\frac{1}{2} K_{2} \xi_{\mathrm{i}, \mathrm{i}} \xi_{\mathrm{i}}+K_{3} \xi E_{\mathrm{ii}} \geqslant 0
$$

\footnotetext{
${ }^{1}$ Here and henceforth the superscript ${ }^{*}$ represents the quantity in the reference configuration.
} 
where $\lambda, \mu$ are Lamé's parameters. Moreover, interface conditions can be imposed at the boundary between bone and material zones, $\partial \mathcal{B}_{i . f}$., by assuming an energy density

$$
\mathscr{E}_{i . f .}=\frac{1}{2} K_{4}\left(\xi^{+}-\xi^{-}\right)^{2}=\frac{1}{2} K_{4}[|\xi|]^{2} \geqslant 0,
$$

where $[|\xi|]$ denotes the jump of $\xi$ through the boundary $\partial \mathcal{B}_{i . f .}$. The assumed conditions of non negativity (6) and (7) may be satisfied by requiring

$$
\begin{aligned}
& \mu \geqslant 0, \quad K_{1} \geqslant 0, \quad K_{2} \geqslant 0, \quad K_{4} \geqslant 0, \\
& 3 \lambda+2 \mu \geqslant 0, \quad(3 \lambda+2 \mu) K_{1} \geqslant 12 K_{3}{ }^{2} .
\end{aligned}
$$

In order to specify mixed displacement-traction boundary value problem in the linear theory of elastic materials with voids the following data must be given:

- the geometry of the body $\mathcal{B}$, the field $\varsigma^{*}\left(\mathbf{X}, t_{0}\right)$, and the material properties $\lambda, \mu, K_{1}, K_{2}, K_{3}$ at the initial time $t_{0}$;

- the body force field $b_{\mathrm{i}}$;

- the surface traction $\tau_{\mathrm{i}}(\mathbf{X}, t)$ specified on $\partial_{\tau} \mathcal{B}$ for the time interval in which the solution is desired $\left[t_{0}, t_{f}\right]$

- the continuous surface displacement $u_{\mathrm{i}}(\mathbf{X}, t)$ specified on the boundary $\partial_{u} \mathcal{B}$ for $\left[t_{0}, t_{f}\right]$;

- the continuous initial displacement field $u_{\mathrm{i}}^{0}(\mathbf{X})$ and the continuous initial change in volume fraction $\xi^{0}(\mathbf{X})$ for all $\mathbf{X} \in \mathcal{B}$.

Note that we are assuming that the elastic properties of the porous solid are given and that the effect of the pores is lumped into the corresponding material constants. Alternatively, these properties could be estimated analytically from the elastic properties of the pure solid, the geometry of the pores and the porosity, following an approach [49] based on Eshelby's inclusion theory [48].

In a mixed boundary-value-problem there is a portion of the boundary on which the displacement are specified and a portion of the boundary on which the surface tractions are specified. These portions of the boundary are denoted by $\partial_{u} \mathcal{B}$ and $\partial_{\tau} \mathcal{B}$, respectively, their union is the entire boundary $\partial \mathcal{B}$. With these data the typical mixed boundary-value problem is to determine the fields $u_{\mathrm{i}}(\boldsymbol{x}, t), \xi(\boldsymbol{x}, t)$, $E_{\mathrm{ij}}(\boldsymbol{x}, t)$, and the Piola stress tensor $T_{\mathrm{ij}}(\boldsymbol{x}, t)$ corresponding to $b_{\mathrm{i}}$, which satisfy the initial conditions

$$
u_{\mathrm{i}}(\boldsymbol{x}, 0)=u_{\mathrm{i}}^{0}(\boldsymbol{x}), \quad \xi(\boldsymbol{x}, 0)=\xi^{0}(\boldsymbol{x}), \quad \forall \boldsymbol{x} \in \mathcal{B},
$$

the displacement boundary condition

$$
u_{\mathrm{i}}(\boldsymbol{x}, t)=\tilde{u}_{\mathrm{i}}(\boldsymbol{x}, t), \quad \forall t \in\left[t_{0}, t_{f}\right], \quad \forall \boldsymbol{x} \in \partial_{u} \mathcal{B},
$$

the surface traction boundary condition

$$
\tau_{\mathrm{i}}(\boldsymbol{x}, t)=T_{\mathrm{ij}}(\boldsymbol{x}, t) n_{j}=\tilde{\tau}_{\mathrm{i}}(\boldsymbol{x}, t), \quad \forall t \in\left[t_{0}, t_{f}\right], \quad \forall \boldsymbol{x} \in \partial_{\tau} \mathcal{B},
$$

where $\boldsymbol{n}$ is the unit vector normal to boundary $\partial_{\tau} \mathcal{B}$, and the condition on the flux of the change of volume fraction $\xi$ on $\partial \mathcal{B}$ is given by

$$
\xi_{, \mathrm{i}}(\boldsymbol{x}, t) n_{\mathrm{i}}=\Xi, \quad \forall t \in\left[t_{0}, t_{f}\right], \quad \forall \boldsymbol{x} \in \partial \mathcal{B},
$$

where $\boldsymbol{n}$ is the unit vector normal to boundary $\partial \mathcal{B}$. In the framework of the weak formulation, we will consider variations in both the partial position $x_{\mathrm{i}}$ and the volume fraction $\varsigma$ associated with the material particle $\mathbf{X}$. A variation performed holding the particle $\mathbf{X}$ fixed will be denoted by $\delta$. We require that the stored energy function $\mathscr{E}$ of a material with voids satisfies, for all variations in $x_{\mathrm{i}}$ and $\varsigma$, the equality

$$
\begin{aligned}
\delta \mathcal{A}=\int_{\mathcal{B}}\left[T_{\mathrm{ij}} \delta E_{\mathrm{ij}}+K_{1} \xi \delta \xi+K_{2} \xi_{, \mathrm{i}} \delta \xi_{, \mathrm{i}}+K_{3}\left(E_{\mathrm{ii}} \delta \xi+\xi \delta E_{\mathrm{ii}}\right)-b_{\mathrm{i}} \delta u_{\mathrm{i}}\right] \mathrm{d} \mathcal{V}+ \\
\quad-\int_{\partial_{\tau} \mathcal{B}} \tau_{\mathrm{i}} \delta u_{\mathrm{i}} \mathrm{d} \mathcal{S}-\int_{\partial \mathcal{B}} \Xi \delta \xi \mathrm{d} \mathcal{S}+\int_{\partial \mathcal{B}_{i . f .}} K_{4}[|\xi|] \delta[|\xi|] \mathrm{d} \mathcal{S}=0,
\end{aligned}
$$

where

$$
T_{\mathrm{ij}}=2 \mu E_{\mathrm{ij}}+\lambda E_{\mathrm{kk}} \delta_{\mathrm{ij}} .
$$


The equation (13) is responsible for the mechanical coupling between displacement $u_{\mathrm{i}}$ and matrix volume fraction $\varsigma$ at each step.

In order to take into account mass changes due to biological activities, the reference matrix volume fraction $\varsigma^{*}$ is not constant, but is assumed to vary in time according to specific growthresorption laws which will be specified in the following.

According to what done in the preceding papers [70, 69], the signal $S$ sent by the osteocytes to the sensor cells is assumed to take the following form

$$
S=\int_{\mathcal{B}} \mathscr{E}\left(\boldsymbol{x}_{0}, t\right) \hat{d}\left(\hat{\rho}_{\mathrm{b}}\left(\boldsymbol{x}_{0}, t\right)\right) e^{-f\left(\boldsymbol{x}-\boldsymbol{x}_{0}\right)} \mathrm{d} \boldsymbol{x}_{0}-P_{\text {ref }}=P(\boldsymbol{x}, t)-P_{\text {ref }},
$$

where $\hat{d}\left(\rho_{\mathrm{b}}\right)=\eta \rho_{\mathrm{b}} / \rho_{\mathrm{b}}^{\mathrm{Max}}$ is the density of active sensor cells, which are assumed present only in living bone-tissue $[60,70,69]$. The attenuation law of the signal was assumed to be the function:

$$
f\left(\boldsymbol{x}-\boldsymbol{x}_{0}\right)=\frac{\left\|\boldsymbol{x}-\boldsymbol{x}_{0}\right\|}{D}
$$

where the length $D$ represents the range of action of sensor cells and the value $P_{\text {ref }}$ is the reference value of stimulus, associated with a biological equilibrium state for which the effect of resorption and synthesis are balanced.

\subsection{Time-dependent evolution rules of growth and resorption}

This section is concerned with the formulation of the evolution rules in bones reconstructed with bio-resorbable materials using the tools of the continuum theory of mixtures. Specifically, we consider a mixture with two constituent: bone living tissue and bio-resorbable material. The reference mass density of the mixture can be expressed as:

$$
\rho^{*}=\rho_{\mathrm{b}}{ }^{*}+\rho_{\mathrm{m}}{ }^{*},
$$

where $\rho_{\mathrm{b}}^{*}$ and $\rho_{\mathrm{m}}^{*}$ are respectively the mass densities of bone and of bio-resorbable material in the reference configuration. Analogously, the volume fraction of solid matrix can be written simply:

$$
\varsigma^{*}=\left(\frac{\rho_{\mathrm{b}}^{*}}{\rho_{\mathrm{b}}^{\operatorname{Max}}}+\frac{\rho_{\mathrm{m}}^{*}}{\rho_{\mathrm{m}}^{\operatorname{Max}}}\right)=\varsigma_{\mathrm{b}}^{*}+\varsigma_{\mathrm{m}}^{*},
$$

being $\rho_{\mathrm{b}}^{\mathrm{Max}}$ the density of cortical bone, which we assume here to be approximately equal to the density of mineralized tissue $\gamma^{*}$, introduced in Eq. (2). Moreover, $\rho_{\mathrm{m}}^{\mathrm{Max}}$ is the mass density of bio-resorbable material without pores. Material properties of the mixture are assumed isotropic, linear elastic and instead of Lamé's parameters $(\lambda$ and $\mu$ ) in Eq. (6), we introduce Young's modulus $Y$ and Poisson's ratio $\nu$. In particular, the elastic modulus $Y$ depends on the reference mass density fields, $\rho_{\mathrm{b}}^{*}$ and $\rho_{\mathrm{m}}^{*}$, of bone tissue and bio-material respectively, according to [27]

$$
Y=Y_{\mathrm{b}}^{\operatorname{Max}}\left(\frac{\rho_{\mathrm{b}}^{*}}{\rho_{\mathrm{b}}^{\operatorname{Max}}}\right)^{\beta_{\mathrm{b}}}+Y_{\mathrm{m}}^{\operatorname{Max}}\left(\frac{\rho_{\mathrm{m}}^{*}}{\rho_{\mathrm{m}}^{\operatorname{Max}}}\right)^{\beta_{\mathrm{m}}}=Y_{\mathrm{b}}^{\operatorname{Max}}\left(\varsigma_{\mathrm{b}}^{*}\right)^{\beta_{\mathrm{b}}}+Y_{\mathrm{m}}^{\operatorname{Max}}\left(\varsigma_{\mathrm{m}}{ }^{*}\right)^{\beta_{\mathrm{m}}},
$$

where the maximal elastic moduli $Y_{\mathrm{b}}^{\mathrm{Max}}, Y_{\mathrm{m}}^{\mathrm{Max}}$ and exponents $\beta_{\mathrm{b}}, \beta_{\mathrm{m}}$ are constants. As a first approximation, we assume that the Poisson's ratio is constant $(\nu=0.3)$ and do not change when the local apparent density changes. The time evolution of reference mass densities of bone tissue and of bioresorbable material is governed by biological activity and thus the mass densities rates are assumed to depend on the local value of the stimulus and on porosity according to the following rules $[60,70,69]$

$$
\left\{\begin{array}{l}
\dot{\rho}_{\mathrm{b}}^{*}=A_{\mathrm{b}}(S) H(\phi) \\
\dot{\rho}_{\mathrm{m}}^{*}=A_{\mathrm{m}}(S) H(\phi)
\end{array} .\right.
$$

It is worth noting for sake of clarity that in Eq. (20), the stimulus $S$ is affected by mechanical causes and establishes, however, a link between mechanical and biological phenomena. In particular, the following forms for the functions $A_{\mathrm{b}}$ and $A_{\mathrm{m}}$ have been chosen to perform numerical simulations:

$$
A_{\mathrm{b}}(S)=\left\{\begin{array}{cc}
s_{\mathrm{b}} S & \text { for } S \geqslant 0 \\
r_{\mathrm{b}} S & \text { for } S<0
\end{array},\right.
$$




$$
A_{\mathrm{m}}(S)=\left\{\begin{array}{ll}
0 & \text { for } S \geqslant 0 \\
r_{\mathrm{m}} S & \text { for } S<0
\end{array},\right.
$$

where the functions $A_{\mathrm{b}}$ and $A_{\mathrm{m}}$ are piece-wise linear functions with different slopes for negative and positive values of stimulus. Parameters $s_{b}, r_{b}$ and $r_{m}$ are positive constants and they represent bone synthesis rate, bone resorption rate and bio-material resorption rate, respectively. In Eq. (20) the current porosity, $\phi$, is considered because osteocytes, i.e. mechanosensory cells of bone, detect what occurs in the instantaneous configuration:

$$
\phi(\boldsymbol{x}, t)=1-\theta\left(\frac{\rho_{\mathrm{b}}(\boldsymbol{x}, t)}{\rho_{\mathrm{b}}^{\operatorname{Max}}}+\frac{\rho_{\mathrm{m}}(\boldsymbol{x}, t)}{\rho_{\mathrm{m}}^{\operatorname{Max}}}\right)=1-\theta\left(\varsigma_{\mathrm{b}}+\varsigma_{\mathrm{m}}\right) \quad 0<\theta \leqslant 1 .
$$

In the mixture, if the actual porosity is too low then living cells will not be able to efficiently resorb the bio-material neither to form new bone tissue, as the available space will not allow for the action of a suitably large number of actor cells. If the porosity, on the other hand, is too large then we do not have enough matter (i.e. bone tissue or bio-material) on which actor cells may deposit. Also in this circumstance remodeling will not occur quickly enough or will not occur at all. The choice for the function $H$ was justified by the intention of matching the biological behavior described above. We choose the shape of $H$ in such a way that $H=0$ for $\phi=0$ or $\phi=1$, namely (see [72, 10] and Fig. 1a):

$$
H=k \phi(1-\phi),
$$

where $k$ is a suitable constant parameter, which was assumed equal to 4 from this point forward. The kinematical constraint that is reasonable in the case of high porosity is the constraint of incompressible distributed volume [53], i.e. the volume associated with the effective mass of the mixture, $\mathcal{V}_{\mathrm{f}}$. In detail, one can show that, passing from the reference configuration to the instantaneous one, an element of distributed volume transforms according to the expression:

$$
d \mathcal{V}_{\mathrm{f}}=\frac{\varsigma}{\varsigma^{*}} J d \mathcal{V}_{\mathrm{f}}^{*}
$$

where $J \simeq 1+\operatorname{tr}(E)$ is the linearized ratio of volumetric expansion. Thus the kinematical constraint can be expressed by the equation

$$
\frac{\varsigma}{\varsigma^{*}} J=1
$$

The same relationship (26), being valid for each constituent of the mixture, implies that

$$
\frac{\varsigma_{\mathrm{b}}}{\varsigma_{\mathrm{b}}^{*}} J=1, \quad \frac{\varsigma_{\mathrm{m}}}{\varsigma_{\mathrm{m}}^{*}} J=1 .
$$

Since the apparent mass densities are also transported by the rule $\rho^{*}=J \rho$, the last equations also imply that the true mass densities of bone tissue and bio-resorbable material are constant with respect to time, respectively $\gamma_{\mathrm{b}}=\gamma_{\mathrm{b}}^{*}$ and $\gamma_{\mathrm{m}}=\gamma_{\mathrm{m}}^{*}$.

As the mixture apparent density changes during remodeling, the material parameter $K_{1}$, is calculated as a power function of the apparent density analogous to that of Young's modulus:

$$
K_{1}=K_{1 \mathrm{~b}}^{\operatorname{Max}}\left(\frac{\rho_{\mathrm{b}}^{*}}{\rho_{\mathrm{b}}^{\operatorname{Max}}}\right)^{\alpha_{\mathrm{b}}}+K_{1 \mathrm{~m}}^{\operatorname{Max}}\left(\frac{\rho_{\mathrm{m}}^{*}}{\rho_{\mathrm{m}}^{\operatorname{Max}}}\right)^{\alpha_{\mathrm{m}}}=K_{1 \mathrm{~b}}^{\operatorname{Max}}\left(\varsigma_{\mathrm{b}}{ }^{*}\right)^{\alpha_{\mathrm{b}}}+K_{1 \mathrm{~m}}^{\operatorname{Max}}\left(\varsigma_{\mathrm{m}}{ }^{*}\right)^{\alpha_{\mathrm{m}}} .
$$

In fact $K_{1}$ can be interpreted as a pore stiffness.

It can be demonstrated (see App. A) that the influence of deformation on the porosity increases as the parameter $\lambda_{v}=K_{3}{ }^{2} / K_{1}$ increases. In order to estimate the parameter $K_{3}$ we hypothesize that $\lambda_{v}$ - a correction of $\lambda$ that accounts for the effect of voids - can vary, depending on the porosity, as a fraction of Lamé parameter $\lambda$. Thus, $K_{3}$ can be expressed by

$$
K_{3}=\sqrt{\hat{g}\left(\phi^{*}\right) \lambda K_{1}}
$$

where the function $\hat{g}\left(\phi^{*}\right)$ non-negative and less than one, is considered in order to maximize the effect of voids when the level of porosity is high and to nullify this effect when porosity tends to zero. We 

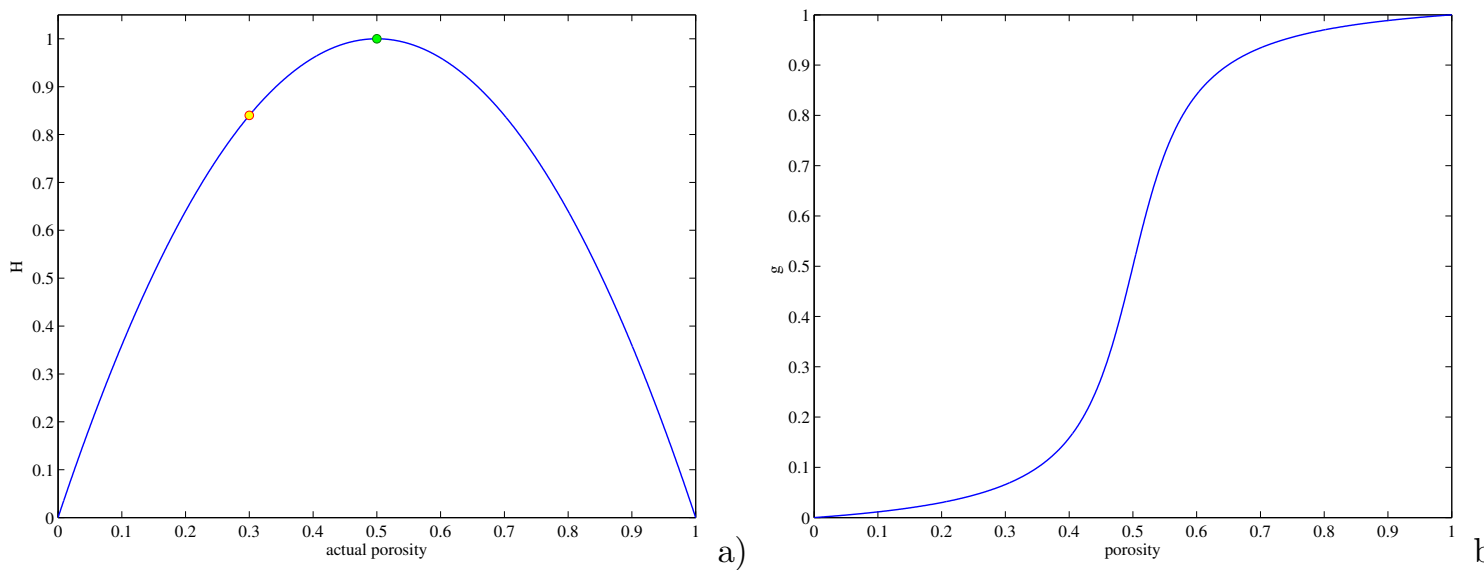

a)

b)

Figure 1. Plot of function $H(\phi)$ a) and $\left.\hat{g}\left(\phi^{*}\right) \mathbf{b}\right)$.

can for instance assume that the function $\hat{g}$ takes the form plotted in Fig. 1b,

$$
\hat{g}\left(\phi^{*}\right)=A_{k_{3}}\left(\frac{1}{\pi} \operatorname{arctg}\left[s_{k_{3}}\left(\phi^{*}-\frac{1}{2}\right)\right]+\frac{1}{2}\right)-k_{03},
$$

in which the parameter $A_{k_{3}}$ affects the amplitude of function $\hat{g}\left(\phi^{*}\right)$ and ranges from 0 to 1 , the parameter $s_{k_{3}}$ has an effect on the slope of the curve and $k_{03}$ is a constant to nullify the value of the function $\hat{g}\left(\phi^{*}\right)$ when porosity vanishes.

One can easily see that $K_{3}=0$ for $\phi^{*}=0$. It is trivial to note that the vanishing of $A_{k_{3}}$ implies the decoupling between the mechanical behavior of the voids and the biological evolution of the mass densities; when the parameter $A_{k_{3}}$ attains the unit value, it follows that $\lambda_{v}=\lambda$, namely $\lambda_{e q}=\lambda-\lambda_{v}=0$ (see App. A): the material with voids retains only the shear stiffness. Therefore, the total stiffness of the material with voids decreases with the increase of $A_{k_{3}}$.

\subsection{Attractor state stimulus}

If the net rate of change of bone apparent density is close to zero, we assume that a 'quasi-equilibrium' condition exists. In this state, we assume that the tissue energy level $P(\boldsymbol{x}, t)$ is approximately equal to a constant, which we call the attractor state energy $P_{\text {ref }}$, i.e., $P \cong P_{\text {ref }}$. The term 'attractor state' refers to the principle that many physical and biological systems tend toward (or are attracted toward) certain target or attractor states, although these states may never actually be reached. The actual state may simply fluctuate around or drift toward the attractor state and create a 'quasi-equilibrium' condition. Near the attractor state, there is little net bone remodeling, so that $\rho_{\mathrm{b}} \cong \rho_{\mathrm{b} \text { ref. It has }}$ been suggested $[10,11]$ the possible existence of multiple regions of stimulus: (a) a central region corresponding to a range of 'normal' activities, where will be an appreciable gain or loss of bone; (b) a region to the left of center corresponding to decreased levels of stimulus; and (c) a region to the right of center corresponding to increased levels of stimulus. For values of stimulus greater than normal, bone growth will result. For values of the stimulus less than normal, bone resorption will occur. The relationship used in the present work can be expressed mathematically as follows:

$$
S(\boldsymbol{x}, t)=\left\{\begin{array}{lr}
P(\boldsymbol{x}, t)-P_{\mathrm{ref}}^{\mathrm{s}} & \text { for } P(\boldsymbol{x}, t)>P_{\mathrm{ref}}^{\mathrm{s}} \\
0 & \text { for } P_{\mathrm{ref}}^{\mathrm{r}} \leqslant P(\boldsymbol{x}, t) \leqslant P_{\text {ref }}^{\mathrm{s}} \\
P(\boldsymbol{x}, t)-P_{\mathrm{ref}}^{\mathrm{r}} & \text { for } P(\boldsymbol{x}, t)<P_{\text {ref }}^{\mathrm{r}}
\end{array} .\right.
$$

In the previous formulas, the apices 's' and ' $\mathrm{r}$ ' stand for synthesis and resorption, respectively. $P_{\text {ref }}^{\mathrm{s}}$ is calculated for $(1+\delta / 100) \varepsilon_{\text {ref }}$, and $P_{\text {ref }}^{\mathrm{r}}$ is calculated for $(1-\delta / 100) \varepsilon_{\text {ref }}$, where $\varepsilon_{\text {ref }}$ is the reference homeostatic strain, i.e. the center of strain state interval, which coincides with the so-called lazy zone, 


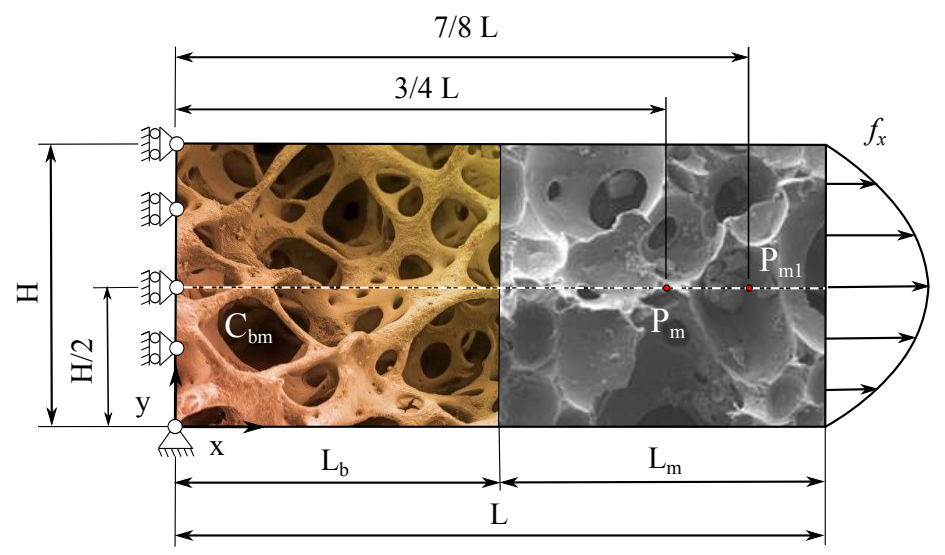

FiguRE 2. Schematic presentation of the model considered in numerical examinations. Interface and its close environment of bone tissue (left) and bone-substitute (right) is presented.

where the osteoregulatory balance of the bone is maintained [91]; the endpoints of the lazy zone are defined by means of the non negative parameter $\delta$.

In this paper the biological signal is assumed to be generated instantaneously by the static value of deformation energy. Actually it is well-known that the resorption and synthesis phenomena show a crucial dependence on the time variations of mechanical loads. What seems significant is the role of micro-porosity and micro-flows induced by cyclic loads, see e.g. [57].

In this context, the models presented here will need to be generalized in order to account for, e.g., i) frequency dependence, ii) memory effects and iii) internal resonance phenomena, which are significant to bone growth and resorption. These generalizations will need to introduce some extra kinematical descriptors in order to account for micro-vibrations induced by externally applied variable loads and for those phenomena of energy distribution, trapping and flow which were studied -in a different context- in the papers $[14,58,23,13]$.

\subsection{Problem in non-dimensional form}

In order to simplify the analysis, a non-dimensional form of the considered problem ${ }^{2}$ is achieved normalizing the mass densities of bone and material with respect to $\rho_{\mathrm{b}}^{\mathrm{Max}}$ :

- mass density of bone, $\tilde{\rho}_{\mathrm{b}}=\rho_{\mathrm{b}} / \rho_{\mathrm{b}}^{\mathrm{Max}}=\varsigma_{\mathrm{b}}$,

- mass density of material, $\tilde{\rho}_{\mathrm{m}}=\rho_{\mathrm{m}} / \rho_{\mathrm{b}}^{\operatorname{Max}}=\left(\rho_{\mathrm{m}}^{\operatorname{Max}} / \rho_{\mathrm{b}}^{\mathrm{Max}}\right) \varsigma_{\mathrm{m}}$,

- total mass density, $\tilde{\rho}=\tilde{\rho}_{\mathrm{b}}+\tilde{\rho}_{\mathrm{m}}$;

Normalizing the Young's moduli of bone and material with respect to $Y_{\mathrm{b}}^{\mathrm{Max}}$ gives non-dimensional

- Young's modulus of bone tissue, $\tilde{Y}_{\mathrm{b}}=Y_{\mathrm{b}} / Y_{\mathrm{b}}^{\mathrm{Max}}$;

- Young's modulus of material, $\tilde{Y}_{\mathrm{m}}=Y_{\mathrm{m}} / Y_{\mathrm{b}}^{\mathrm{Max}}$.

The Young's modulus of the mixture varies with mass density according to Eq. (19); thus, the corresponding dimensionless Young's modulus takes the form:

$$
\tilde{Y}=\left(\varsigma_{\mathrm{b}}^{*}\right)^{\beta_{\mathrm{b}}}+\tilde{Y}_{\mathrm{m}}^{\operatorname{Max}}\left(\varsigma_{\mathrm{m}}^{*}\right)^{\beta_{\mathrm{m}}},
$$

where $\beta_{\mathrm{b}}$ and $\beta_{\mathrm{m}}$ are constants (see [27]), and $\tilde{Y}_{\mathrm{b}}^{\mathrm{Max}}=1, \tilde{Y}_{\mathrm{m}}^{\mathrm{Max}}=Y_{\mathrm{m}}^{\mathrm{Max}} / Y_{\mathrm{b}}^{\mathrm{Max}}$ and $\tilde{Y}=Y / Y_{\mathrm{b}}^{\mathrm{Max}}$. The stored energy density $\mathscr{E}$, Eq. (6), will be normalized with respect to the maximum value of the Young's modulus $Y_{\mathrm{b}}^{\mathrm{Max}}$ :

$$
\tilde{\mathscr{E}}=\mathscr{E} / Y_{\mathrm{b}}^{\mathrm{Max}}
$$

\footnotetext{
${ }^{2}$ The quantities in non-dimensional form are denoted by a superimposed tilde.
} 
As a consequence of this normalization, the material parameters involved in the Eq. (6) and used to describe the behavior of the change of matrix volume fraction and its derivatives become in a dimensionless form:

$$
\begin{gathered}
\tilde{K}_{1}=K_{1} / Y_{\mathrm{b}}^{\mathrm{Max}}, \quad \tilde{K}_{2}=K_{2} /\left(Y_{\mathrm{b}}^{\mathrm{Max}} L_{0}^{2}\right), \\
\tilde{K}_{3}=K_{3} / Y_{\mathrm{b}}^{\mathrm{Max}}, \quad \tilde{K}_{4}=K_{4} / Y_{\mathrm{b}}^{\mathrm{Max}}, \\
\tilde{\lambda}_{\mathrm{eq}}=\lambda_{\mathrm{eq}} / Y_{\mathrm{b}}^{\mathrm{Max}}, \quad \tilde{\lambda}=\lambda / Y_{\mathrm{b}}^{\mathrm{Max}}, \quad \tilde{\lambda}_{v}=\lambda_{v} / Y_{\mathrm{b}}^{\mathrm{Max}},
\end{gathered}
$$

with $L_{0}$ a characteristic length; in the following analyses $L_{0}$ is assumed to be equal to the length of the sample. The non-dimensional stimulus can be defined as:

$$
\tilde{S}=\tilde{P}-\tilde{P}_{\text {ref }}
$$

where $\tilde{P}_{\text {ref }}=P_{\text {ref }} /\left(Y_{\mathrm{b}}^{\mathrm{Max}} L_{0}^{3}\right)$ and $\tilde{P}$ comes from the assumption (33) and the Eq. (15). It is worth noting that in the case of simplified 2-D geometry the dimensionless Stimulus is defined replacing $L_{0}^{3}$ with $L_{0}^{2}$.

A characteristic time $t_{\text {ref }}$ can be interpreted as the order of magnitude (e.g. months) of the time duration in which the physiological processes that lead to fulfillment of the phenomenon of material resorption and bone synthesis are accomplished. This characteristic time allows introducing

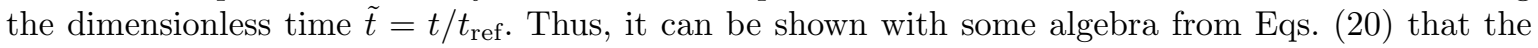
dimensionless form of the evolution equations for the mass densities is:

$$
\left\{\begin{array}{c}
\frac{\partial \tilde{\rho}_{\mathrm{b}}}{\partial \tilde{t}}=\tilde{A}_{\mathrm{b}}(\tilde{S}) H(\phi) \\
\frac{\partial \tilde{\rho}_{\mathrm{m}}}{\partial \tilde{t}}=\tilde{A}_{\mathrm{m}}(\tilde{S}) H(\phi)
\end{array},\right.
$$

where starting from Eqs. (21) and (22) one gets:

$$
\begin{gathered}
\tilde{A}_{\mathrm{b}}(\tilde{S})=\left\{\begin{array}{ll}
\tilde{s}_{\mathrm{b}} \tilde{S} & \text { for } \tilde{S} \geqslant 0 \\
\tilde{r}_{\mathrm{b}} \tilde{S} & \text { for } \tilde{S}<0
\end{array},\right. \\
\tilde{A}_{\mathrm{m}}(\tilde{S})=\left\{\begin{array}{ll}
0 & \text { for } \tilde{S} \geqslant 0 \\
\tilde{r}_{\mathrm{m}} \tilde{S} & \text { for } \tilde{S}<0
\end{array},\right.
\end{gathered}
$$

with

$$
\tilde{s}_{\mathrm{b}}=\frac{Y_{\mathrm{b}}^{\mathrm{Max}} L_{0}^{3} t_{\mathrm{ref}}}{\rho_{\mathrm{b}}^{\mathrm{Max}}} s_{\mathrm{b}} ; \quad \tilde{r}_{\mathrm{b}}=\frac{Y_{\mathrm{b}}^{\mathrm{Max}} L_{0}^{3} t_{\mathrm{ref}}}{\rho_{\mathrm{b}}^{\mathrm{Max}}} r_{\mathrm{b}} ; \quad \tilde{r}_{\mathrm{m}}=\frac{Y_{\mathrm{b}}^{\mathrm{Max}} L_{0}^{3} t_{\mathrm{ref}}}{\rho_{\mathrm{b}}^{\mathrm{Max}}} r_{\mathrm{m}} .
$$

Also for dimensionless rates of resorption and synthesis, $L_{0}^{3}$ is to substitute with $L_{0}^{2}$ in the case of simplified 2-D geometry. According to the results reported in [80], the characteristic time $t_{\text {ref }}=$ $9.7 \times 10^{7} \mathrm{~s}$ can be estimated in order to have a duration for the whole process of approximately a hundred days.

\section{Results}

\subsection{Generalities}

A sample is considered which behaves as a membrane, composed by two materials, under the plain stress assumption and with elastic modulus and porosity variable with respect to time and space. The sequence of configurations of the considered bone-material sample were calculated via the automatic code COMSOL Multiphysics. The sample is considered of length $L$ with initially completely separated bone tissue and bio-material, hence assuming that one part of the slab is initially occupied by pure bone tissue and the remaining part is occupied by pure bio-material. As far the sizes of the specimen were concerned, it was assumed $L_{\mathrm{b}}=L_{\mathrm{m}}=1.25 \mathrm{~mm}, L=L_{\mathrm{b}}+L_{\mathrm{m}}$, the height $H=H_{\mathrm{b}}=H_{\mathrm{m}}=1.00 \mathrm{~mm}$ and the thickness of the specimen equal to half of the height $H$. As for boundary conditions, the slab was constrained at $x=0\left(u_{1}(0, y, t)=0 \forall y, u_{2}(0,0, t)=0 ; \forall t\right)$. An external load of lineic density $f_{x}$ having parabolic distribution was applied at $x=L$, as shown in Fig. 2; a reference load amplitude 
TABLE 1. Values of the parameters used in numerical simulations.

\begin{tabular}{lcccccccc}
\hline$\rho_{\mathrm{b}}^{\operatorname{Max}}=\rho_{\mathrm{m}}^{\operatorname{Max}}$ & $Y_{\mathrm{b}}^{\operatorname{Max}}=Y_{\mathrm{m}}^{\mathrm{Max}}$ & $\beta_{\mathrm{b}}=\beta_{\mathrm{m}}$ & $k$ & $\theta$ & $\eta$ & $D$ & $\varepsilon_{\mathrm{ref}}$ & $P_{\text {ref }}$ \\
\hline $1800 \mathrm{~kg} / \mathrm{m}^{3}$ & $14.0 \mathrm{GPa}$ & 2.5 & 4 & 1.0 & 1.0 & $0.1 L$ & $10^{-3}$ & $3.47 \times 10^{-4} \mathrm{~J}$ \\
\hline
\end{tabular}

TABLE 2. Values of the material coefficients used in numerical simulations.

\begin{tabular}{ccccccc}
\hline$K_{1 \mathrm{~b}}^{\mathrm{Max}}=K_{1 \mathrm{~m}}^{\mathrm{Max}}$ & $\alpha_{\mathrm{b}}=\alpha_{\mathrm{m}}$ & $K_{2}$ & $K_{4}$ & $s_{\mathrm{b}}$ & $r_{\mathrm{b}}$ & $r_{\mathrm{m}}$ \\
\hline $11.8 \mathrm{GPa}$ & 2.5 & $10^{-1} \mathrm{~N}$ & $10^{4} \mathrm{~Pa}$ & $1.03 \mathrm{~s} / \mathrm{m}^{4}$ & $1.03 \mathrm{~s} / \mathrm{m}^{4}$ & $0.517 \mathrm{~s} / \mathrm{m}^{4}$ \\
\hline
\end{tabular}

$f_{x \text { ref }}=1.43 \times 10^{7} \mathrm{~N} / \mathrm{m}$ was assumed to normalize the external load. In the presentation which follows, our approach to solve for the distribution of bone and bio-material morphology is to consider the two constituents of the mixture as initially isotropic and homogeneous in which apparent densities and material parameters may subsequently vary as a function of position as remodeling of the bone and resorption of bio-material proceed. The values of the parameters used in the performed numerical simulations are summarized in tables 1 and 2; the corresponding values in on-dimensional form are reported in the table 3 . In particular the influence distance $D=L / 10$ was assumed equal to the thickness of five layers of osteocytes from the bone surface (and micro-venues). In order to consider a potential mesh refinement the size of the smallest region of influence should be determined which fits the biological behavior. Thus, the mesh size was chosen as a suitable fraction $(1 / 4)$ of the influence distance $D$, to accomplish a numerical integration sufficiently accurate for the problem at hand. Time duration was set equal to 0.15 time units. At the beginning of the process $(t=0)$, the initial values of mass densities were assumed - unless otherwise stated - to be $\rho_{\mathrm{b}}^{0}=\rho_{\mathrm{m}}^{0}=900 \mathrm{~kg} / \mathrm{m}^{3}$, and therefore Young's moduli, according to Eq. (19), became: $Y_{\mathrm{b}}^{0}=Y_{\mathrm{m}}^{0}=2.5 \mathrm{GPa}$.

Spatial distributions and time evolutions of mass densities of bone and material, of change of matrix volume fraction and of porosity of mixture were evaluated at each instant by means of the incremental procedure outlined in section 2; the results were presented by showing (1-D) spatial distributions at the end of the process along the longitudinal sampling line $C_{\mathrm{bm}}:\{x \in[0, L], y=$ $H / 2\}$ through the whole domain, and time evolutions in the probe points $P_{\mathrm{m}} \equiv(3 / 4 L, H / 2)$, and $P_{\mathrm{m} 1} \equiv(7 / 8 L, H / 2)$ (see Fig. 2). In the presented figures, the convention of indicating with an arrow the direction of the increase of involved parameters, was adopted to facilitate understanding by the reader.

The width of the lazy zone $P_{\text {ref }}^{\mathrm{s}}-P_{\text {ref }}^{\mathrm{r}}$, Eq. (31), was assumed to be in accordance with the value $\delta=5$ in the following numerical simulations. The quantities plotted in the following figures are referred to the actual configuration of the specimen and appear in their dimensionless form, according to the definitions given in subsection 2.4, unless otherwise stated. In the following numerical simulations, the values $0.1,0.4,0.9$ were assigned to the parameter $A_{k 3}$ which imply an increasing coupling between the mechanical behavior of the voids and the biological evolution of the mass densities. Moreover, with reference to Eq. (30), we assigned the value $s_{k 3}=10$.

\subsection{Code description}

The evolutionary problem in our case study involves time-dependent differential equations of first order for mass densities of bone tissue and bio-material coupled with PDE governing mechanical equilibrium. The evolution of mass densities both, of bone tissue and bio-material, is due to a pure mechanical cause depending on the change of volume fraction, $\xi$, and to a biological cause depending on the action of actor cells. The solver employs a discrete time step increment algorithm based on the backward differentiation formula (BDF) method and uses a Newton-type iterative method to solve this nonlinear systems of PDE's. Since the integro-differential equations we are integrating are strongly non-linear, a very delicate issue in the performed numerical simulations regards the choice of time step 
TABLE 3. Material coefficients in non-dimensional form used in numerical simulations.

\begin{tabular}{ccccccc}
\hline$\tilde{\rho}_{\mathrm{b}}^{0}=\tilde{\rho}_{\mathrm{m}}^{0}$ & $\tilde{K}_{1 \mathrm{~b}}^{\mathrm{Max}}=\tilde{K}_{1 \mathrm{~m}}^{\mathrm{Max}}$ & $\tilde{K}_{2}$ & $\tilde{K}_{4}$ & $\tilde{s}_{\mathrm{b}}$ & $\tilde{r}_{\mathrm{b}}$ & $\tilde{r}_{\mathrm{m}}$ \\
\hline 0.5 & 0.843 & $1.14 \times 10^{-5}$ & $7.14 \times 10^{-6}$ & $1.21 \times 10^{7}$ & $1.21 \times 10^{7}$ & $6.09 \times 10^{6}$ \\
\hline
\end{tabular}

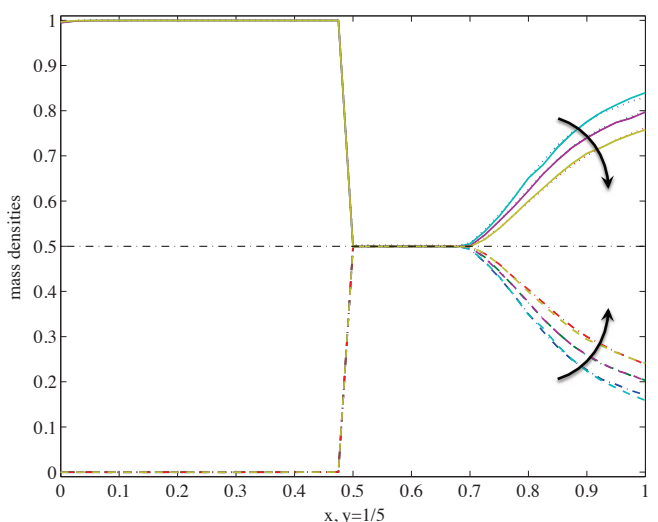

a)

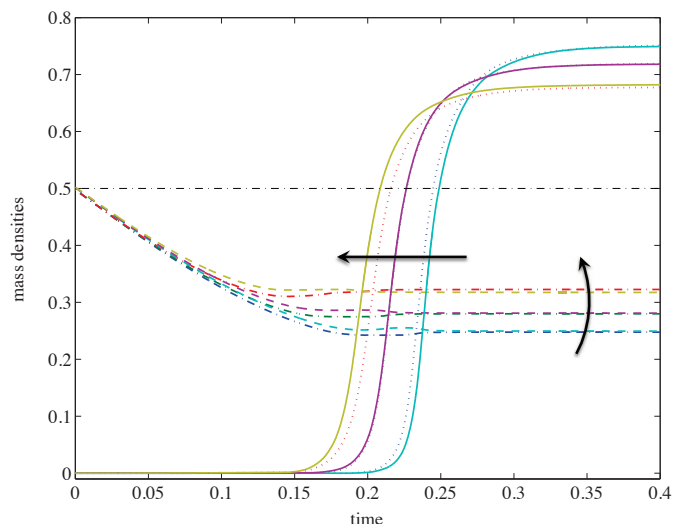

b)

Figure 3. Dimensionless mass densities of bone (solid line for compression, dotted line for tension) and material (dashed line for compression, dot-dashed line for tension): 1-D final distributions along $C_{\mathrm{bm}}$ a) and time evolutions in $\left.P_{\mathrm{m} 1} \mathbf{b}\right)$. The arrows denote the direction of increasing $A_{\mathrm{k} 3}$.

that in our case is variable and optimized. The algorithm employed for solving the evolution equations takes into account some numerical difficulties as reported in [17, 18, 50].

\subsection{Influence of the mechanical behavior of the voids}

3.3.1. Initial porosities of bone and material equal to $50 \%$. In this section we present the results of some numerical calculations aimed at highlighting the role played by the parameter $A_{k_{3}}$ in the case $\rho_{\mathrm{b}}^{0}=\rho_{\mathrm{m}}^{0}=0.5 \rho^{\mathrm{Max}}$; it modulates the amplitude of the curve described by Eq. (30), which adjusts the influence of referential porosity $\phi^{*}$ on the parameter $K_{3}$; stiffness $K_{3}$ in turn governs the coupling between elasticity and porosity, Eq. (6). The green spot in Fig. 1a shows the value of the function $H$, Eq. (24), corresponding to the initial value of porosity.

Figure 3a shows the spatial distributions of the mass densities of bone and material along $C_{\mathrm{bm}}$ at the end of the remodeling process. In the zone of material near the side where the load is applied, the curves of bone and material approach with increasing $A_{k_{3}}$; the maximum difference between the two limit situations (maximum and minimum coupling) amounts to approximately $10 \%$ both for bone and material. No appreciable difference between the behavior in tension and compression is noticed. Figure $3 \mathrm{~b}$ shows the time evolutions of the mass densities of the bone and of the material in $P_{\mathrm{m}}$. In the earlier stage of the process, bone growth is anticipated with increasing $A_{k_{3}}$, to the extent of $35 \%$ compared to the transient range, when comparing the extreme values of the parameter. In the second stage, the actual bone mass density stands at a decreasing value with increasing $A_{k_{3}}$. The mass density of the material increases with increasing $A_{k_{3}}$. In the time evolution of the mass densities of bone and material, one can observe a different behavior depending on whether the state of stress is tensile or compressive. In particular, in the first part of the process relative to the bone, for values of $A_{k_{3}}$ greater than 0.4 (high coupling between elastic deformation and change of matrix volume fraction $\xi$ ), the compression exerts an accelerating action on the phenomenon of bone growth, while the same role is played by the tension for values of $A_{k_{3}}$ less than 0.4 (low coupling between elastic deformation and change of matrix volume fraction $\xi$ ). At the value $A_{k_{3}}=0.4$, the influences of tension and compression 


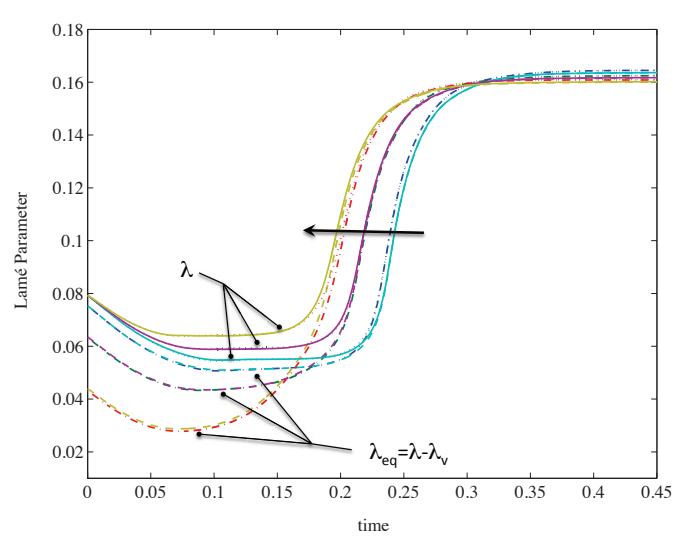

a)

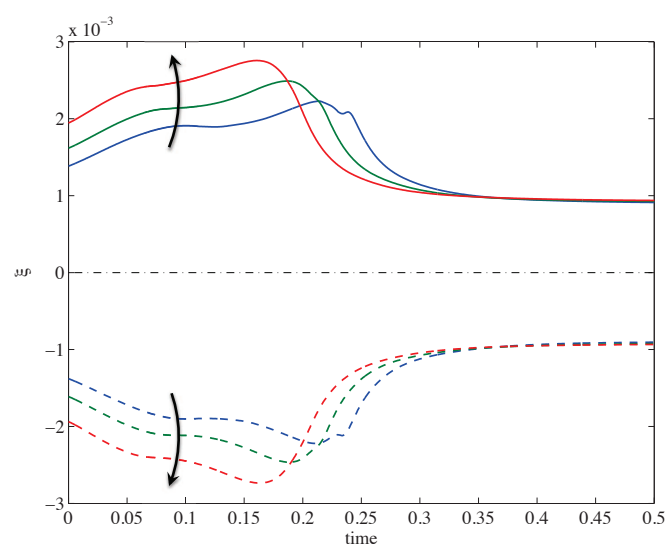

b)

Figure 4. Time evolutions of Lamé parameters $\lambda$ (solid line for compression and dotted line for tension) and $\lambda_{\text {eq }}$ (dashed line for compression and dot-dashed line for tension) in $P_{\mathrm{m}} \mathbf{a}$ ). Time evolutions of the matrix volume fraction change $\xi$ (solid line for compression and dashed line for tension) in $P_{\mathrm{m}} \mathbf{b}$ ). The arrows denote the direction of increasing $A_{\mathrm{k} 3}$.

are balanced almost perfectly, as well as in the situation of the regime. Concerning the evolution of the mass density of the material, it can be observed that in the first part of the process, when the mass density of bone is still low enough, a difference is manifested, albeit modest, between tensile and compressive behavior, the mass density of the material in the case of tension resulting less than that in the case of compression. The difference between the two states of stress is not appreciable in the stationary state.

The influence of the change of matrix volume fraction $\xi$ is manifested by the anticipation of the moment when the growth of bone in the zone of the material begins.

Figure $4 \mathrm{a}$ shows the time evolutions of the Lamé parameter $\lambda$ and of the equivalent Lamé parameter $\lambda_{\text {eq }}$ (see App. A) of the mixture as the parameter $A_{k_{3}}$ varies. With increasing $A_{k_{3}}$, for very low mass density of the bone and in the first part of the process, the difference between $\lambda$ and $\lambda_{\text {eq }}$ increases and a significant reduction of stiffness of the mixture with voids occurs, the maximum value of which is estimated at approximately more than fifty percent. In the second part of the transient, an increase of $A_{k_{3}}$ anticipates the increase phase for the parameters, until the differences are significantly attenuated in the steady state. Also the difference between tension and compression does not seem to be remarkable during the entire duration of the process.

Figure $4 \mathrm{~b}$ shows the time evolutions of the change of matrix volume fraction $\xi$ as the parameter $A_{k_{3}}$ varies. In the first part of the process, with increasing $A_{k_{3}}$ the $\xi$ increases in compression, while decreases in tension. In a second phase, the opposite happens, and finally in the steady-state $\xi$ stands at stationary values, positive in compression and negative in tension. At the end of the process, $\xi$ remains different from zero: the voids remained dilated or contracted, depending on tension or compression. Specifically, it is worth noting that the application of the load, in the case of compression, induces a decrease of the pore volume with respect to the reference configuration. This effect results in an increase of the change of matrix volume fraction, $\xi$. At the end of the process, in the current configuration, the complete saturation of the pores was achieved and then if the load is released, the porosity increases ( $\xi$ decreases). Therefore the positive $\xi$ which is shown in Fig. $4 \mathrm{~b}$ at the end of the process can be interpreted as a measure of the amount of bone, in sense of volume fraction, that has not been formed for the occlusion of the pores due to the load. In the case of tension, instead, the application of the load induces an increase in the volume of the pores with respect to the reference configuration. This expansion results in a reduction of $\xi$. At the end of the process (see Fig. 4b) in the current configuration 


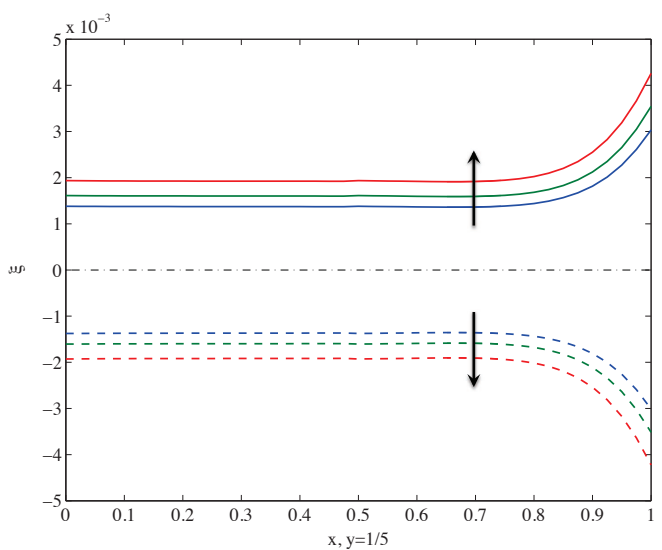

a)

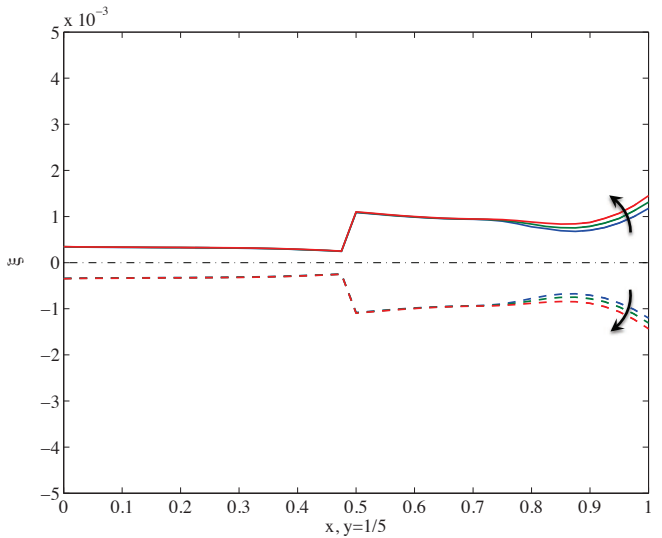

b)

Figure 5. 1-D distributions of the matrix volume fraction change $\xi$ along $C_{\mathrm{bm}}$ under tension (dashed line) and compression (solid line): initial a) and final b) states. The arrows denote the direction of increasing $A_{\mathrm{k} 3}$.

a state of full saturation is established $(\phi=0)$ and therefore the negative $\xi$ can be seen as a measure of the exceeding amount of bone that was formed due to the dilating volume of the pores as a result of the application of the load. The above observations consent to affirm that the addition of the variable $\xi$ in the model allows to better estimate the loss of resistance. Not taking this into account could lead to an underestimation of the risk of failure. For instance, the difference between the mass densities of the bone in the steady-state for $A_{k_{3}}=0.1$ and $A_{k_{3}}=0.9$ is about $10 \%$ (see Fig. 3), which involves a reduction of about $20 \%$ in terms of failure strength of the bone tissue [15].

Figure 5a shows the time evolutions of the change of the matrix volume fraction $\xi$ under tension and compression in the earlier time range of the process as the parameter $A_{k_{3}}$ varies. It is noted that for low values of the bone density, the $\xi$ complaints a greater sensitivity to changes in the parameter $A_{k_{3}}$.

Figure $5 \mathrm{~b}$ shows the distributions of the change of matrix volume fraction $\xi$ along $C_{\mathrm{bm}}$ under tension and compression at the end of the process, as the parameter $A_{k_{3}}$ varies. Also this plot shows that at the end of the process a significant increase (decrease) in $\xi$ in compression (tension) is still remaining, both in the bone and material zones. We observe a discontinuity at the interface, Eq. (7), due to the fact that a soft constraint $\left(K_{4}=10^{+4} \mathrm{~Pa}\right)$ was put on $\xi$ at the interface; in the area of the material close to the side of application of the load, local effects due to the particular distribution of the load itself are observed; in particular $\xi$ increases (decreases) with increasing $A_{k_{3}}$ (denoted by the direction of the arrows) under compression (tension).

Figure 6 shows the time evolutions of the porosity $\phi$ of the mixture as the parameter $A_{k_{3}}$ varies, in terms of both tension and compression. The decrease of $\phi$ is anticipated the greater the value of $A_{k_{3}}$. For $A_{k_{3}}=0.1$, tension causes the decrease of the porosity to occur in advance, while for $A_{k_{3}}=0.9$ this role is played by compression, even if the differences are small.

Figure 7 shows the 2-D distribution of the dimensionless strain energy density under tension $\left(A_{k_{3}}=0.9\right)$, at the beginning of the process. Figure 8 shows initial 2-D distributions of the energy density terms $\tilde{\mathscr{E}}$ under tension $\left(A_{k_{3}}=0.9\right)$ associated with the parameter $K_{1}$ (see Eq. (28) and table 2), accounting for the direct effect of the voids (Fig. 8a), and $K_{3}$ (see Eq. (29)), accounting for the coupling between the change of volume fraction $\xi$ and the elastic deformation $E$ (Fig. $8 \mathrm{~b}$ ), within the bi-dimensional domain of the sample.

3.3.2. Initial porosities of bone and material equal to $\mathbf{3 0 \%}$. The following numerical simulations relate to the case in which the initial situation of the sample is characterized by mass densities of bone and material $\tilde{\rho}_{\mathrm{b}}^{0}=\tilde{\rho}_{\mathrm{m}}^{0}=0.7$, and then $\phi^{0}=0.3$ (Eq. (23) and yellow spot in Fig. 1a). In this way a 


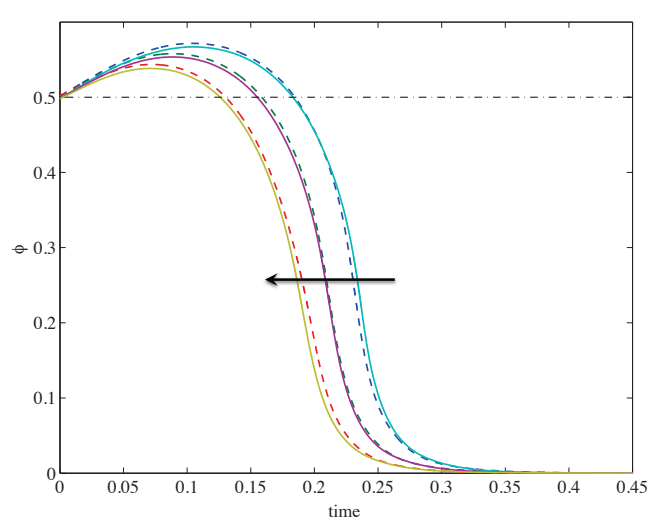

Figure 6. Time evolutions of the porosity under compression (solid line) and tension (dashed line) in $P_{\mathrm{m}}$. The arrow denotes the direction of increasing $A_{\mathrm{k} 3}$.

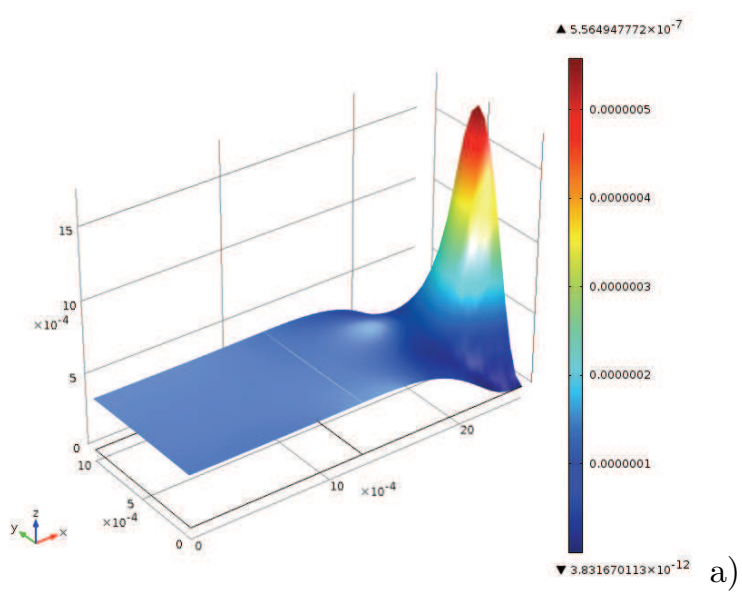

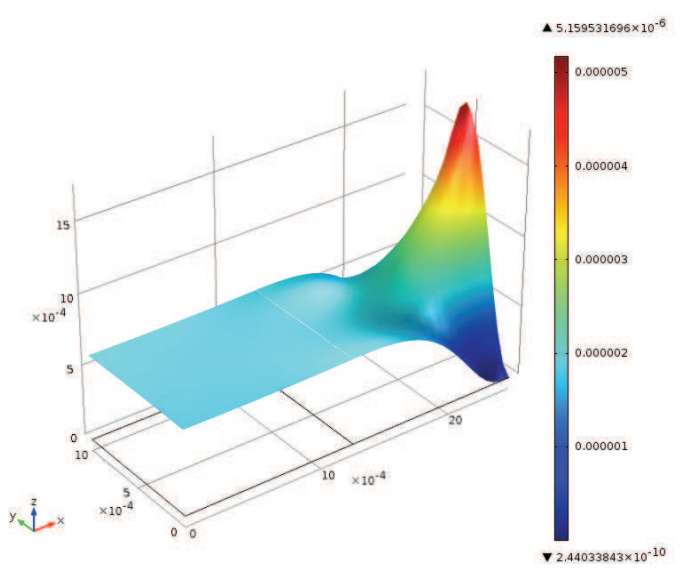

FiguRE 7. 2-D distributions of the dimensionless Strain energy density under tension $\left(A_{k_{3}}=0.9\right)$.

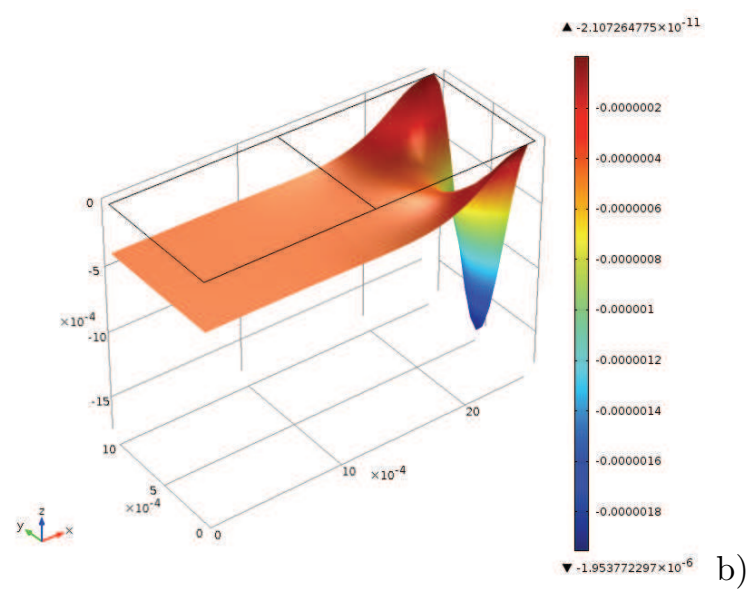

Figure 8. 2-D distributions of the energy density terms in $\tilde{\mathscr{E}}$ under tension $\left(A_{k_{3}}=\right.$ $0.9)$ associated with the parameters $K_{1}$ a) and $K_{3} \mathbf{b}$ )

starting point is chosen departing from which possible significant changes of the function $H$, Eq. (24) and Figure 1b, are made possible. As far as the external load is concerned, a magnification factor equal to 1.75 is applied with respect to the reference load amplitude $f_{x r e f}$ adopted in subsection 3.3.1. In this case, attention was paid only to the extreme values $A_{k 3}=0.1$ and $A_{k 3}=0.9$ for brevity's sake. Figure 9a shows the spatial distributions of the mass densities of the bone and of the material along $C_{\mathrm{bm}}$ at the end of the process. It may be noted that in the first half of the zone of the material, the mass density of the material clearly prevails over that of the bone and the influence of the parameter $A_{k_{3}}$ can not be noticed, while the opposite happens in the second half; in particular, the curves tend to approach as $A_{k_{3}}$ is increasing. It may be noted that in the portion of the material closest to the side of load application, the curves get closer as $A_{k_{3}}$ augments; the maximum difference between the two limit situations (maximum and minimum coupling) is attained along the side where the load is applied and amounts to about $18 \%$ both for bone and material. The value of $A_{k_{3}}$ being the same, the inner curves are to refer to traction, and obviously the outer ones to compression. 


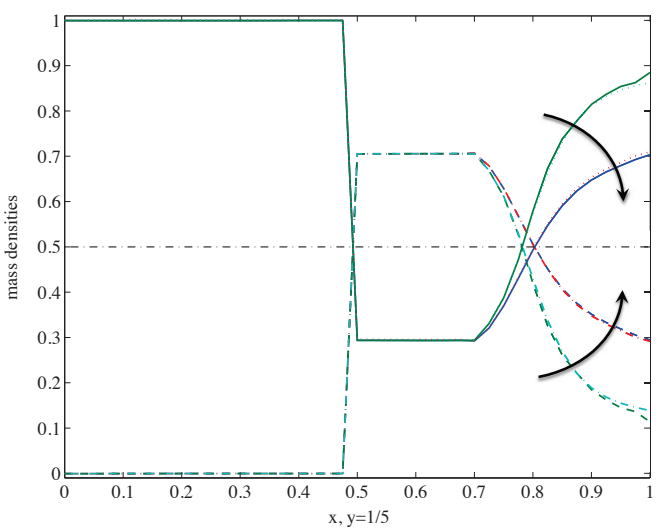

a)

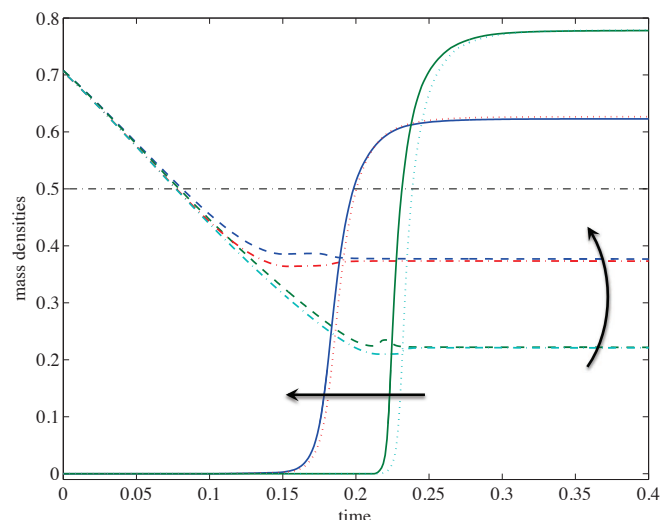

b)

Figure 9. Dimensionless mass densities of bone (solid line for compression, dotted line for tension) and material (dashed line for compression, dot-dashed line for tension): 1-D final distributions along $C_{\mathrm{bm}}$ a) and time evolutions in $\left.P_{\mathrm{m} 1} \mathbf{b}\right)$. The arrows denote the direction of increasing $A_{\mathrm{k} 3}$.

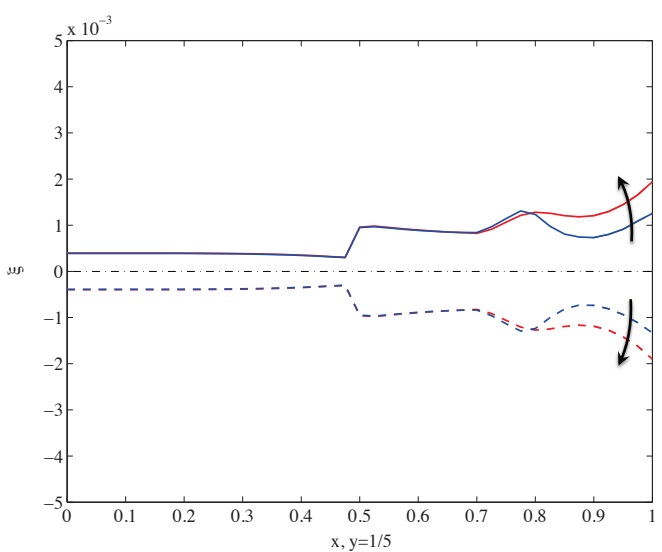

a)

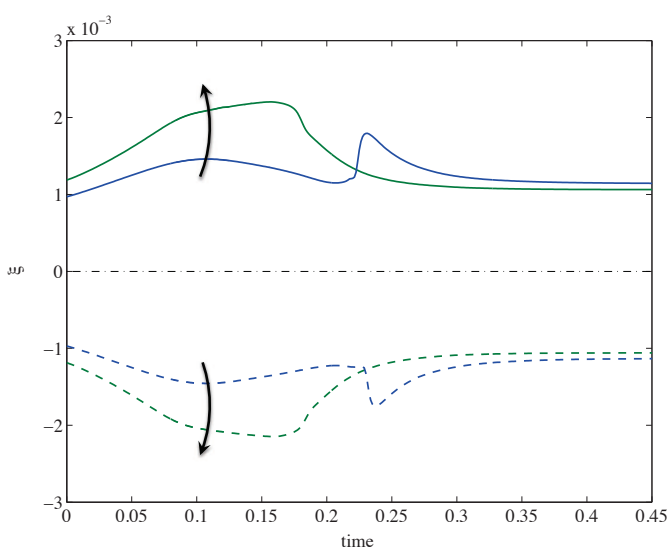

b)

Figure 10. Change of matrix volume fraction $\xi$ under tension (dashed line) and compression (solid line): 1-D final distributions along $C_{\mathrm{bm}}$ a) and time evolutions in $\left.P_{\mathrm{m}} \mathbf{b}\right)$. The arrows denote the direction of increasing $A_{\mathrm{k} 3}$.

Figure $9 \mathrm{~b}$ shows the time evolutions of the mass densities of the bone and of the material in $P_{\mathrm{m} 1}$. It can be observed that the bone growth is anticipated as $A_{k_{3}}$ increases, the maximum difference being recorded at the extreme values of the parameter, to the extent of $41 \%$. The attainment of the stationary state by the material is anticipated as $A_{k_{3}}$ increases. In the steady state, the stationary value of the bone density is lower the higher is $A_{k_{3}}$, while the opposite happens for the material; the value of $A_{k_{3}}$ being equal, compression anticipates bone growth and leads to a larger stationary density. Regarding the material, always $A_{k_{3}}$ being equal, compression in a first transitional phase leads to density values greater than those induced by traction, then the effect is reversed in the steady state. Figure 10a shows the spatial evolutions of the change of the matrix volume fraction $\xi$ along $C_{\mathrm{bm}}$ at the end of the process. It can be noted that the $\xi$ takes positive values in compression, negative in traction; near the side of load application the influence of the parameter $A_{k_{3}}$ clearly manifests: its reduction causes a decrease of $\xi$ in compression and its increase in traction. 


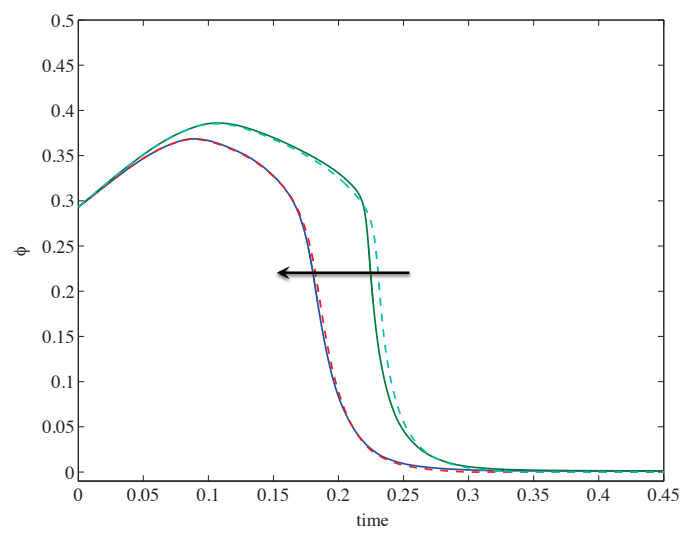

a)

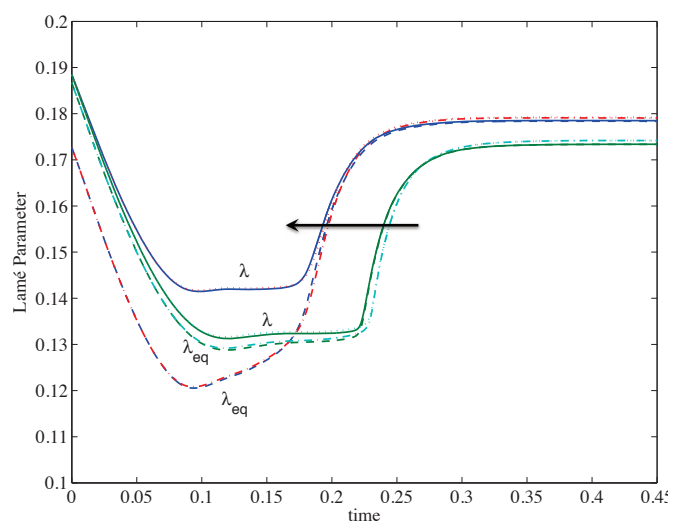

b)

Figure 11. Time evolutions of the porosity in $P_{\mathrm{m}}$ under tension (dashed line) and compression (solid line) a); time evolutions of Lamé parameters $\lambda$ (solid line for compression and dotted line for tension) and $\lambda_{\text {eq }}$ (dashed line for compression and dot-dashed line for tension) in $P_{\mathrm{m}} \mathbf{b}$ ). The arrows denote the direction of increasing $A_{\mathrm{k} 3}$.

Figure 10b shows the time evolutions of $\xi$ in $P_{\mathrm{m}}$. Even in this plot, compression determines the positive values of $\xi$, negative traction. In the process transient, the increase of $A_{k_{3}}$ causes an increase of $\xi$ under compression and a decrease under tension, while in the steady state the opposite happens even if less significantly. Figure 11a shows the time evolutions of the porosity $\phi$ in $P_{\mathrm{m}}$. In the second half of the transient phase, the increase of $A_{k_{3}}$ causes an anticipation in the decay of $\phi$; thus the curve corresponding to $A_{k 3}=0.9$ remains at lower level with respect to the curve for $A_{k 3}=0.1$. Compression also contributes, albeit to a lesser extent, in this effect. In the steady state, any difference disappears because the saturation is achieved in any case.

Figure 11b shows the time evolutions of the parameter $\lambda$ and of the equivalent parameter $\lambda_{\text {eq }}$ as the parameter $A_{k_{3}}$ varies. At the beginning of the process the decrement of $\lambda_{\text {eq }}$ with respect to $\lambda$ is about $8 \%$ and $1 \%$ for $A_{k 3}=0.9$ and $A_{k 3}=0.1$ respectively. In the transient state the maximum decrement of $\lambda_{\text {eq }}$ with respect to $\lambda$ is about $15 \%$ and $2 \%$ for $A_{k 3}=0.9$ and $A_{k 3}=0.1$ respectively. In the steady state, the values of $\lambda$ and $\lambda_{\text {eq }}$ realign, remaining $5 \%\left(A_{k_{3}}=0.9\right)$ and $7.5 \%\left(A_{k_{3}}=0.1\right)$ below the initial value of $\lambda$. This lead us to remark that a considerable risk exists which is related to an underestimation of the deformation capacity of the sample due to the lack of consideration of the presence of voids and to the coupling of this mechanical phenomenon with biological evolution. Figure 12 shows the spatial distribution of the stimulus at the beginning (a) and end (b) of the process. It may be noted that the stimulus at the end is not null but the process still ends because the porosity was completely filled and therefore there is no more space for bone accretion.

3.3.3. Different maximum mass densities of bone and biomaterial. We will now analyze a realistic situation in which a mechanical mass density lower than that of the bone tissue has been assigned to the bio-resorbable material, for the fact that preference may be given to focus on other characteristics (e.g. stiffness, rate of resorption, biocompatibility etc.) in the design of the biomaterial and hence to accept some difference as far as other aspects are concerned for instance mass density. Therefore, in the present study, the following maximum

- $\rho_{\mathrm{b}}^{\mathrm{Max}}=1800 \mathrm{~kg} / \mathrm{m}^{3}$,

- $\rho_{\mathrm{m}}^{\mathrm{Max}}=1400 \mathrm{~kg} / \mathrm{m}^{3}$,

and initial values $\rho_{\mathrm{b}}^{0}=\rho_{\mathrm{b}}^{\operatorname{Max}} / 2$ and $\rho_{\mathrm{m}}^{0}=\rho_{\mathrm{m}}^{\mathrm{Max}} / 2$ are assumed for mass densities of bone and material respectively. As far as the external load is concerned, an magnification factor equal to 1.3 is applied 

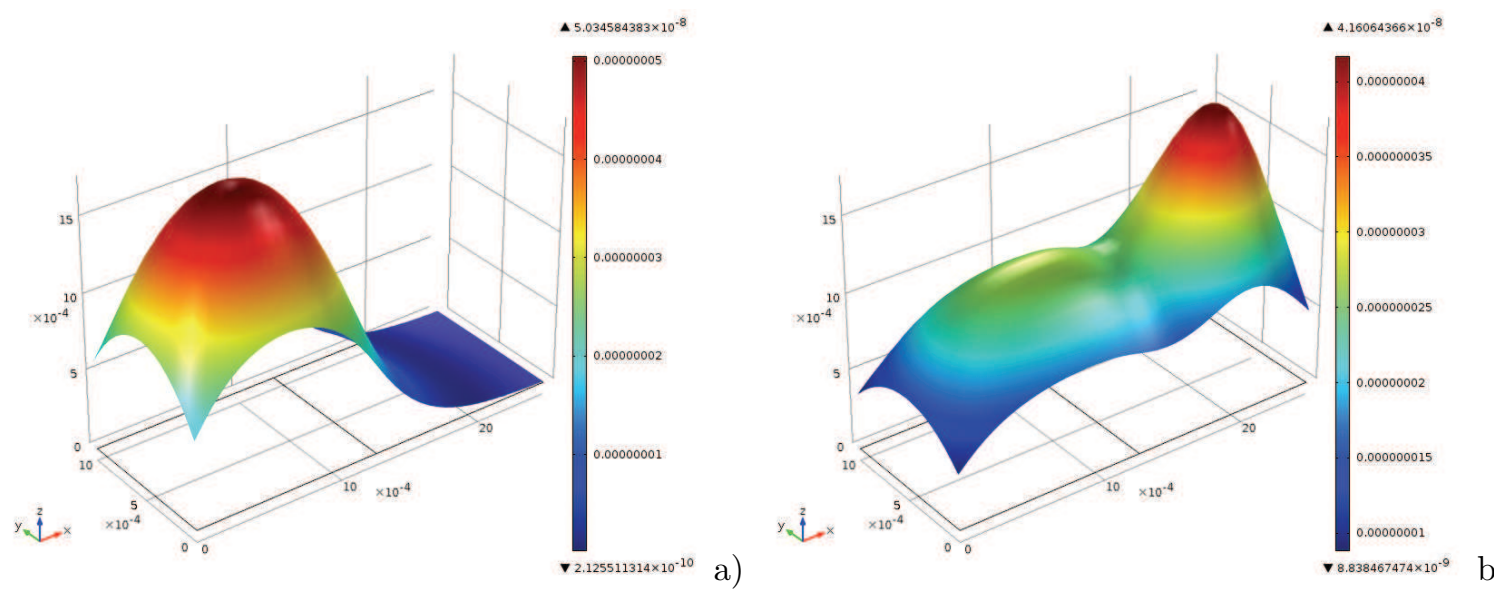

Figure 12. Initial a) and final b) 2-D distributions of stimulus $\tilde{S}$ under tension $\left(A_{k_{3}}=0.9\right)$.
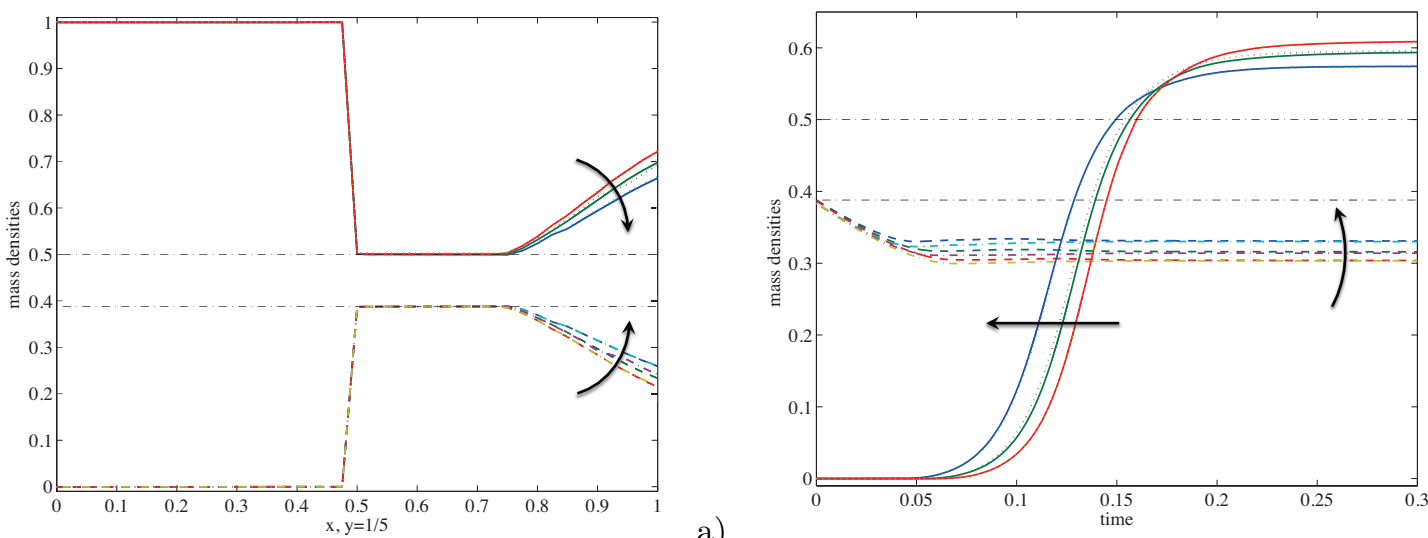

a)

b)

Figure 13. Dimensionless mass densities of bone (solid line for compression, dotted line for tension) and material (dashed line for compression, dot-dashed line for tension): 1-D final distributions along $C_{\mathrm{bm}}$ a) and time evolutions in $P_{\mathrm{m} 1} \mathbf{b}$ ). The arrows denote the direction of increasing $A_{\mathrm{k} 3}$.

with respect to the reference load amplitude $f_{x r e f}$ adopted in subsection 3.3.1. Figure 13a shows the spatial distributions of the mass densities of bone (solid line for compression, dotted line for tension) and material (dashed line for compression, dot-dashed line for tension) along $C_{\mathrm{bm}}$ at the end of the process. In the zone of material near the side where the load is applied, the curves of bone and material approach with increasing. The two extreme situations $A_{k 3}=0.1$ and $A_{k 3}=0.9$ differ by about $5 \%$ in terms of mass density at the edge of load application, both for bone and material. This involves a local reduction of about $10 \%$ of their failure strength, according to the formula proposed in [15]. No appreciable difference between the behavior in tension and compression is noticed.

Figure 13b shows the time evolutions of the mass densities of the bone and of the material in $P_{\mathrm{m} 1}$. In the earlier range of the process, bone growth is anticipated with increasing $A_{k 3}$, while in the second stage, the bone mass density stands at a decreasing value with increasing $A_{k 3}$. The mass density of the material increases as $A_{k 3}$ increases. In the time evolution of the mass densities of bone and material, one can observe that, as far as the influence of the state of stress is concerned in the first part of the process relative to the bone, for values of $A_{k 3}$ approximately equal to 0.4 (middle coupling between 


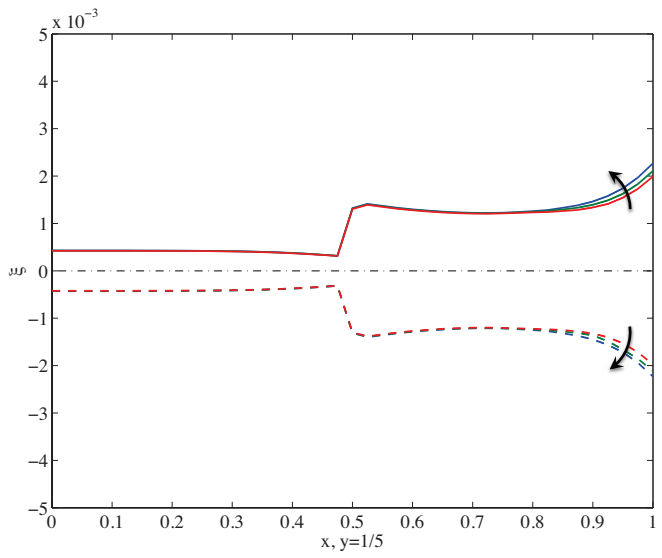

a)

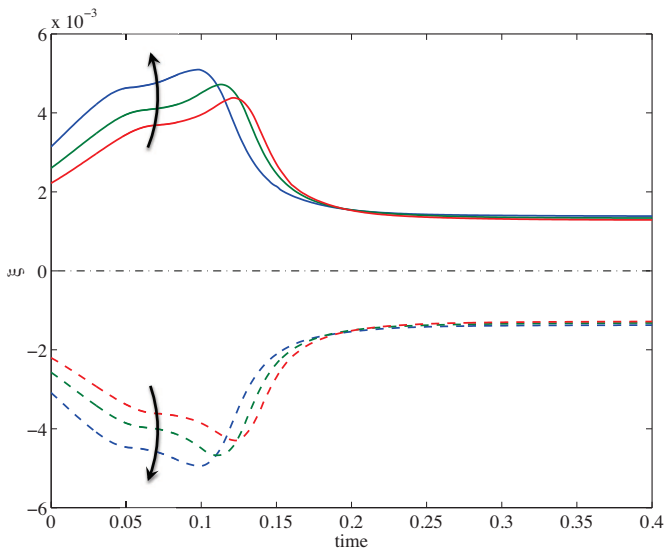

b)

Figure 14. Changes of matrix volume fraction $\xi$ under tension (dashed line) and compression (solid line): 1-D final distributions along $C_{\mathrm{bm}}$ a); time evolutions in $P_{\mathrm{m} 1}$ b). The arrows denote the direction of increasing $A_{\mathrm{k} 3}$.

elastic deformation and change of matrix volume fraction $\xi$ ), the compression exerts an accelerating action on the phenomenon of bone growth, while the influences of tension and compression are balanced almost perfectly in the situation of the regime. Concerning the evolution of the mass density of the material, it can be observed that in the first part of the process, when the mass density of bone is still low enough, a difference appears between tensile and compressive behavior, the mass density of the material in the case of compression resulting slightly larger than that in the case of tension. The difference between the two states of stress is less appreciable in the stationary state. The effect of increment of $A_{k 3}$ is manifested by the anticipation (about 12\%) of the instant when the growth of bone in the zone of the material begins with respect to the transient range by comparison of extreme values of $A_{k 3}$. Figure 14a shows the distributions of the change of matrix volume fraction $\xi$ along $C_{\mathrm{bm}}$ under tension and compression at the end of the process, as the parameter $A_{k 3}$ varies. Also this plot shows that at the end of the process a significant increase (decrease) in $\xi$ in compression (tension) is still remaining, both in the bone and material zones. We observe a discontinuity at the interface, Eq. (7), due to the fact that a soft constraint $\left(K_{4}=10^{4} \mathrm{~Pa}\right)$ was put on $\xi$ at the interface; in the area of the material close to the side of application of the load, local effects due to the particular distribution of the load itself are observed; in particular $\xi$ increases (decreases) with increasing $A_{k 3}$ under compression (tension). Figure 14b shows the time evolutions of the change of matrix volume fraction $\xi$ in $P_{\mathrm{m} 1}$ as the parameter $A_{k 3}$ varies. In the first part of the process, with increasing $A_{k 3}$ the $\xi$ increases in compression, while decreases in tension. In a second phase, the opposite happens, and finally in the steady-state $\xi$ stands at stationary values, positive in compression and negative in tension. At the end of the process, $\xi$ remains different from zero: the voids remained dilated or contracted, depending on tension or compression.

Figure $15 \mathrm{a}$ shows the time evolutions of the porosity of the mixture as the parameter $A_{k 3}$ varies, in terms of both tension and compression. The decrease of $\phi$ is anticipated the greater the value of $A_{k 3}$; moreover, the compression causes the decrease of the porosity to be anticipated for $A_{k 3}=0.9$; such an effect is visible only in first part of the transient range for $A_{k 3}=0.1$; this behavior is inverted moving from the first part to the second part of the transitional interval, even if the differences are small. Figure $15 \mathrm{~b}$ shows the time evolutions of Lamé $\lambda$ and $\lambda_{\text {eq }}$ parameters of the mixture in $P_{\mathrm{m} 1}$ as the parameter $A_{k 3}$ varies. Recalling that $\lambda$ and $\lambda_{\text {eq }}$ represent a measure of the mixture deformability without and with voids, the comparison between $\lambda$ and $\lambda_{\text {eq }}$ is performed in correspondence of the value 0.9 of the parameter $A_{k 3}$, which corresponds to the maximum influence of the mechanical behavior of the voids on the biological evolution of the mass densities. It is noted that at the beginning of 

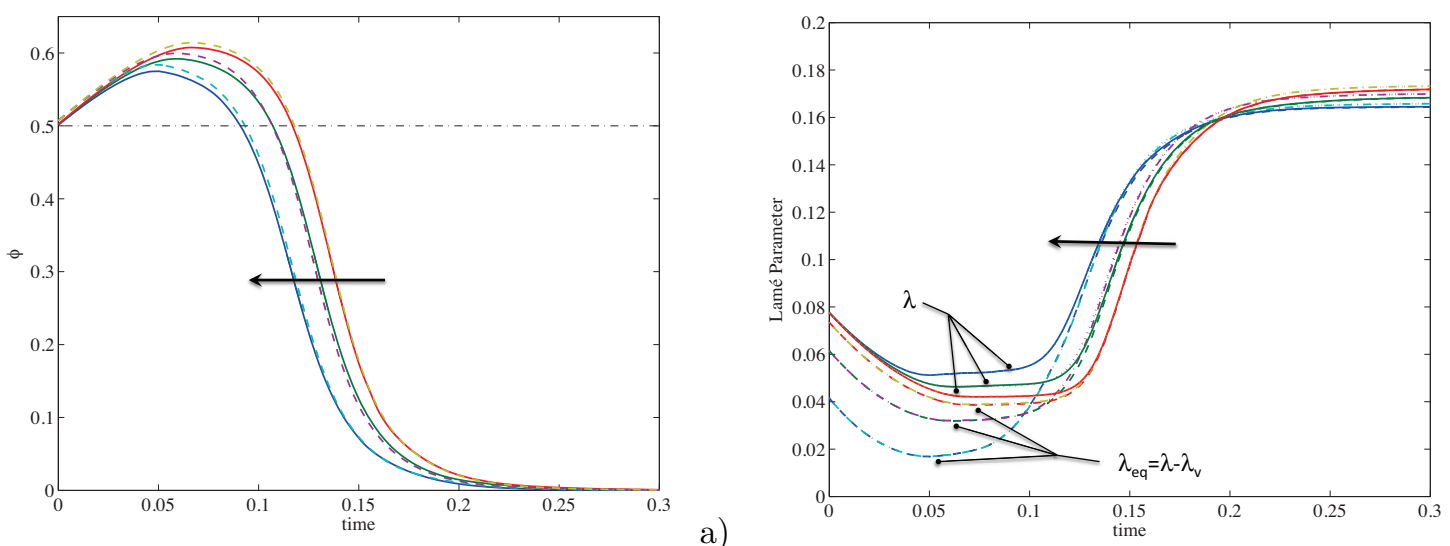

b)

Figure 15. Time evolutions in $P_{\mathrm{m} 1}$ of the porosity (dashed line for tension and solid line for compression) a); and of Lamé $\lambda$ (solid line for compression and dotted line for tension) and $\lambda_{\text {eq }}$ (dashed line for compression and dot-dashed line for tension) parameters of the mixture b). The arrows denote the direction of increasing $A_{\mathrm{k} 3}$.

the process there is a decrease of approximately $46 \%$ of $\lambda_{\text {eq }}$ with respect to $\lambda$; in the region of the transient range, where $\lambda$ and $\lambda_{\text {eq }}$ attain their minimum, the increase in the deformability of the mixture is significantly influenced by the increase in $A_{k 3}$ if account is taken of the presence of voids, since $\lambda_{\mathrm{eq}}$ is about $68 \%$ less than $\lambda$. In the steady state, the values of $\lambda$ and $\lambda_{\text {eq }}$ realign, exceeding of $124 \%\left(A_{k 3}=0.1\right)$ and $113 \%\left(A_{k 3}=0.9\right)$ the common value of $\lambda$ at the beginning of the process. The behaviors in tension and compression do not significantly differ. One can repeat here considerations similar to those already made in the preceding subsections 3.3.1 and 3.3.2 concerning the risks associated with significant reductions in the stiffness of the mixture, albeit under spatially and temporally limited conditions.

\section{Discussion and conclusions}

Small defects in bone tissue can be easily treated by bone itself, due to its self-healing property. However, in the case of extensive bone defects, this self-healing mechanism for the reconstruction of large bone segments is not feasible. In recent years, synthetic grafts have been developed as substitutions of bone grafts. Ideally, a scaffold must be bioresorbable and possess adequate mechanical properties suited to the biological site. Both scaffold and bone exhibit a solid structure characterized by voids that plays a key role in assisting tissue regeneration. Thus, in this paper a mixture model of bone tissue and bioresorbalbe material with evolving mass densities is proposed to describe bone tissue synthesis and resorption when a bio-resorbable material of the kind used in bone reconstruction is present. In particular the theory for the behavior of porous solids was employed in which the matrix material is linearly elastic and the interstices are void of material.

Numerical simulations were worked out which provided ranges of the bio-mechanical parameters for which residual regions of bio-materials remain in remodeled bone and when almost all bio-material is completely replaced by bone tissue. Spatial distributions and time evolutions of mass densities of bone and bio-material and of porosity of mixture were evaluated at each instant; the results were reported by showing spatial distributions at the end of the process along sampling lines and time evolutions in probe points. The novelty presented in this paper was two-folds: a) applying the linear theory of elastic materials with voids, formulated by Cowin and Nunziato [19], to account for the mechanical influence of voids on the mechano-biological evolution of a mixture comprised of bone tissue and bio-resorbable material under mechanical stimulus; and b) coupling the above mentioned static theory with the time evolution formulated by dell'Isola, Lekszycki and Madeo [60, 70]. In 
TABLE 4. Decreases of mass densities and failure strengths.

\begin{tabular}{cccc}
\hline & \multicolumn{2}{c}{$\rho_{\mathrm{b}}^{\mathrm{Max}}=\rho_{\mathrm{m}}^{\mathrm{Max}}$} & $\rho_{\mathrm{m}}^{\mathrm{Max}}=7 / 9 \rho_{\mathrm{b}}^{\operatorname{Max}}$ \\
\hline & $\phi_{\mathrm{b}}^{0}=\phi_{\mathrm{m}}^{0}=50 \%$ & $\phi_{\mathrm{b}}^{0}=\phi_{\mathrm{m}}^{0}=30 \%$ & $\phi_{\mathrm{b}}^{0}=\phi_{\mathrm{m}}^{0}=50 \%$ \\
\hline$\Delta \rho / \rho \%$ & 10 & 18 & 5 \\
\hline$\Delta \sigma_{u} / \sigma_{u} \%$ & 19 & 32 & 10 \\
\hline
\end{tabular}

particular, the variation of the volume of the voids is caused by the strain state under external loading conditions. Thus, the achieved result was to formulate a model and to develop a method in order to accomplish the previously described goals.

In order to illustrate the potentialities of the model and of the method proposed in realistic clinical cases, some situations particularly interesting from the application point of view have been taken into consideration

The simulations performed in the present paper consent to affirm that the assumption of the change of matrix volume fraction as an independent variable in the model allows to better estimate the loss of resistance due to the presence of voids inside the solid matrix. Not taking this into account, i.e. disregarding the influence of the mechanical behavior of voids on the biological evolution, could lead to an underestimation of the risk of failure in the mixture during the process of functional adaptation somewhere in the considered domain. For instance, in the zone close to the side where the external load is applied, the difference between the mass densities of the bone in the steady state for the extreme levels of coupling is approximately $10,18,5 \%$ in the cases $\left(\phi_{\mathrm{b}}^{0}=\phi_{\mathrm{m}}^{0}=50 \%, \rho_{\mathrm{b}}^{\operatorname{Max}}=\rho_{\mathrm{m}}^{\operatorname{Max}}\right)$, $\left(\phi_{\mathrm{b}}^{0}=\phi_{\mathrm{m}}^{0}=30 \%, \rho_{\mathrm{b}}^{\operatorname{Max}}=\rho_{\mathrm{m}}^{\operatorname{Max}}\right),\left(\phi_{\mathrm{b}}^{0}=\phi_{\mathrm{m}}^{0}=50 \%, \rho_{\mathrm{m}}^{\mathrm{Max}}=7 / 9 \rho_{\mathrm{b}}^{\operatorname{Max}}\right)$ respectively. Such significant reductions, even if attained only locally and temporarily, will involve a not negligible decrease of about 19, 32, $10 \%$ in terms of failure strength of the bone tissue. Furthermore, with the increase of coupling, in some spatial zones and in some time ranges, the difference between $\lambda$ and $\lambda_{\text {eq }}$ increases and a significant reduction of stiffness of the mixture with voids occurs. A remark analogous to that one already made just above about the failure strength can be done about the risks related to an underestimation of the deformability of the sample, even during the transitional interval and a little less markedly in the stationary one, due to the lack of consideration of the presence of voids and to the coupling of this mechanical phenomenon with biological evolution.

For reader's convenience the main results were resumed in table 4, which reports in a synthetic manner the per cent decreases of mass densities and failure strengths of the mixture, due to the consideration of voids presence, at the maximum level of coupling $\left(A_{k 3}=0.9\right)$.

The authors would like to suggest further developments which in their opinion seem to be necessary to produce the needed effective tool for clinical practice; these developments should include the consideration of the effects related to the flow of interstitial fluid, the production and the diffusion of a hormone-like chemical messenger responsible for transmitting the signal that regulates the stimulus to growth, modeling multiple scales of both bone and biomaterial and the consideration of large deformation effects; in particular, some of the time scales can be identified in the time variation of the external loads, diffusion of hormone-like messenger, progress of the phenomenon of remodeling, and migration of precursor cells. In this regard, one can not fail to mention the difficulties to be dealt with when one wants to model cyclic loading effects on bone remodeling, due to the two sharply different time scales pertaining to the two above mentioned processes.

The results obtained by the above sensitivity analyses allowed to evaluate the influence of the different relevant parameters on the phenomena of growth and resorption of bone tissue and resorption of bioresorbable bio-material. These results could be useful to develop an optimal design of artificial grafts and to guide the choice and preparation of experimental tests. 


\section{Acknowledgment}

The authors wish to express their gratitude to Francesco dell'Isola for his helpful suggestions and comments, and interesting discussions concerning the research in this topic.

\section{Appendix A. A note on the parameter $\lambda_{v}=K_{3}^{2} / K_{1}$}

The expression of energy to displacement and porosity reads as (see Eq. (6)):

$$
\mathscr{E}(E, \xi, \nabla \xi)=\frac{1}{2} \mathbb{C} E \cdot E+\frac{1}{2} K_{1} \xi^{2}+\frac{1}{2} K_{2}|\nabla \xi|^{2}+K_{3} \xi \operatorname{tr}(E) .
$$

Assuming $K_{2}$ to be zero in Eq. (39) yields:

$$
\mathscr{E}(E, \xi)=\frac{1}{2} \mathbb{C} E \cdot E+\frac{1}{2} K_{1} \xi^{2}+K_{3} \xi \operatorname{tr}(E) .
$$

The principle of local equilibrium states that finding $(\boldsymbol{u}, \xi)$ such that

$$
\min \left\{\int_{\mathcal{B}} \mathscr{E}(E, \xi) \mathrm{d} \mathcal{V}\right\}
$$

is equivalent to find $(\boldsymbol{u})$ such that

$$
\min \left\{\int_{\mathcal{B}} \mathscr{E}(E, \hat{f}(E)) \mathrm{d} \mathcal{V}\right\}
$$

where $\hat{f}(E)$ is given by the following equation:

$$
\left.\frac{\partial \mathscr{E}}{\partial \xi}\right|_{\xi=\hat{f}(E)}=K_{1} \hat{f}(E)+K_{3} \operatorname{tr}(E)=0 .
$$

Solving Eq. (42) in terms of $\hat{f}(E)$ gives

$$
\hat{f}(E)=-\frac{K_{3}}{K_{1}} \operatorname{tr}(E) .
$$

Recalling the expression of the energy for linearly elastic isotropic materials

$$
\frac{1}{2} \mathbb{C} E \cdot E=\frac{1}{2}\left\{2 \mu E \cdot E+\lambda[\operatorname{tr}(E)]^{2}\right\}
$$

and substituting Eq. (42) in Eq. (40) lead to a suggestive form of the energy:

$$
\begin{aligned}
\mathscr{E}(E, \xi)=\frac{1}{2}\{2 \mu E \cdot E+ & \left.\lambda[\operatorname{tr}(E)]^{2}\right\}+\frac{1}{2} K_{1}\left[-\frac{K_{3}}{K_{1}} \operatorname{tr}(E)\right]^{2}+K_{3}\left[-\frac{K_{3}}{K_{1}} \operatorname{tr}(E)\right] \operatorname{tr}(E)= \\
& =\frac{1}{2}\left\{2 \mu E \cdot E+\left[\lambda-\frac{\left(K_{3}\right)^{2}}{K_{1}}\right][\operatorname{tr}(E)]^{2}\right\}=\frac{1}{2}\left\{2 \mu E \cdot E+\lambda_{\text {eq }}[\operatorname{tr}(E)]^{2}\right\}
\end{aligned}
$$

where

$$
\lambda_{\mathrm{eq}}=\left[\lambda-\frac{\left(K_{3}\right)^{2}}{K_{1}}\right]=\lambda-\lambda_{v}
$$

represents for the material with voids an equivalent Lamé's first parameter which accounts for the reduction due to porosity. 


\section{References}

[1] U. Andreaus, M. Colloca and D. Iacoviello, Optimal bone density distributions: Numerical analysis of the osteocyte spatial influence in bone remodeling, Computer Methods and Programs in Biomedicine 113(1) (2014), 80-91.

[2] U. Andreaus, I. Giorgio and T. Lekszycki, A 2-D continuum model of a mixture of bone tissue and bioresorbable material for simulating mass density redistribution under load slowly variable in time, J. Applied Math and Mech. (ZAMM) (2013), 1-23, DOI 10.1002/zamm.201200182.

[3] U. Andreaus, M. Colloca and D. Iacoviello, Modelling of trabecular architecture as result of an optimal control procedure, Chapter II. In: Biomedical Imaging and Computational Modeling in Biomechanics (eds. U. Andreaus and D. Iacoviello), Springer, (2012) 19-37.

[4] U. Andreaus, M. Colloca and D. Iacoviello, An optimal control procedure for bone adaptation under mechanical stimulus, Control Eng Pract. 20 (2012), 575-583.

[5] U. Andreaus, M. Colloca, D. Iacoviello and M. Pignataro, Optimal-tuning pid control of adaptive materials for structural efficiency, Struct. Multidiscip. O. 43 (2011), 43-59.

[6] U. Andreaus and M. Colloca, Prediction of micromotion initiation of an implanted femur under physiological loads and constraints using the finite element method, Procs. of the Inst. of Mechanical Engineers. Part H, J. of Engineering in Medicine, 223 (2009), 589-605.

[7] U. Andreaus, M. Colloca and A. Toscano, Mechanical behaviour of a prosthesized human femur: a comparative analysis between walking and stair climbing by using the finite element method, Biophisics and Bioengineering Letters 1 (2008), 1-15.

[8] U. Andreaus, F. dell'Isola and M. Porfiri, Piezoelectric passive distributed controllers for beam flexural vibrations, J. Vib. Control 10 (2004), 625-659.

[9] I. Armentano, M. Dottori, E. Fortunati, S. Mattioli and J. M. Kenny, Biodegradable polymer matrix nanocomposites for tissue engineering: A review, Polymer Degradation and Stability 95 (2010), 2126-2146.

[10] G.S. Beaupré, T.E. Orr and D.R. Carter, An approach for time-dependent bone modelling and remodelling-Theoretical development, J. Orthopaed. Res. 8 (1990), 651-661.

[11] G.S. Beaupré, T.E. Orr and D.R. Carter, An approach for time-dependent bone modelling and remodelling-application: a preliminary remodelling simulation, J. Orthopaed. Res. 8 (1990), 662-670.

[12] A. Carcaterra, Theoretical modelling and simulations of a neuron-based micro-motor, ENOC 2011, in proceedings of the 7th European Nonlinear Oscillations Conference, July 24-29, 2011, Roma.

[13] A. Carcaterra, A. Akay, Transient energy exchange between a primary structure and a set of oscillators: Return time and apparent damping, Journal of the Acoustical Society of America 115(2) (2004), 683-696.

[14] A. Carcaterra, An entropy formulation for the analysis of energy flow between mechanical resonators, Mechanical Systems and Signal Processing 16(5) (2002), 905-920.

[15] D. R. Carter and W. C. Hayes, The compressive behaviour of bone as a two-phase porous structure, J Bone Joint Surg Am. 59 (1977), 954-962.

[16] R. Casanova, D. Moukoko, M. Pithioux, C. Pailler-Mattéi, H. Zahouani and P. Chabrand, Temporal evolution of skeletal regenerated tissue: what can mechanical investigation add to biological?, Med Biol Eng Comput 48 (2010), 811-819.

[17] A. Cazzani and P. Ruge, Numerical aspects of coupling strongly frequency-dependent soil-foundation models with structural finite elements in the time-domain, Soil Dynamics and Earthquake Engineering, 37 (2012), 56-72.

[18] A. Cazzani and C. Lovadina, On some mixed finite element methods for plane membrane problems, Computational Mechanics 20(6) (1997), 560-572.

[19] S. C. Cowin and J. W. Nunziato, Linear elastic materials with voids, J. Elasticity 13 (1983), 125-147.

[20] S. C. Cowin and M. A. Goodman, A Variational Principle for Granular Materials, J. Applied Math and Mech. (ZAMM) 56 (1976), 281-286.

[21] L. Contrafatto and M. Cuomo, A globally convergent numerical algorithm for damaging elasto-plasticity based on the Multiplier method, International Journal for Numerical Methods in Engineering 63(8) (2005), 1089-1125.

[22] R. Contro, C. Poggi and A. Cazzani, Numerical Analysis of Fire Effects on Beam Structures, Engineering Computations (Swansea, Wales), 5(1) (1988), 53-58. 
[23] A. Culla, A. Sestieri, A. Carcaterra, Energy flow uncertainties in vibrating systems: Definition of a statistical confidence factor, Mechanical Systems and Signal Processing 17(3) (2003), 635-663.

[24] M. Cuomo and A. Nicolosi, A poroplastic model for hygro-chemo-mechanical damage of concrete, Computational Modelling of Concrete Structures - Proceedings of EURO-C (2006), 533-542.

[25] M. Cuomo and L. Contrafatto, Stress rate formulation for elastoplastic models with internal variables based on augmented Lagrangian regularisation, International Journal of Solids and Structures 37(29) (2000), 3935-3964.

[26] M. Cuomo, G. Ventura, Complementary energy approach to contact problems based on consistent augmented Lagrangian formulation, Mathematical and Computer Modelling 28(4-8) (1998), 185-204.

[27] J. D. Currey, The effect of porosity and mineral content on the Young's modulus of elasticity of compact bone, J. Biomechanics 21 (1988), 131-139.

[28] F. dell'Isola, A. Madeo and L. Placidi, Linear plane wave propagation and normal transmission and reflection at discontinuity surfaces in second gradient 3D continua, ZAMM Zeitschrift für Angewandte Mathematik und Mechanik 92(1) (2011), 52-71.

[29] F. dell'Isola, A. Madeo and P. Seppecher, Boundary conditions at fluid-permeable interfaces in porous media: A variational approach, International Journal of Solids and Structures 46(17) (2009), 3150-3164.

[30] F. dell'Isola and R. Batra, Saint-Venant's problem for porous linear elastic materials, J. Elasticity 47 (1997), 73-81.

[31] F. dell'Isola and C. Woźniak, On continuum modelling the interphase layers in certain two-phase elastic solids, ZAMM Zeitschrift für Angewandte Mathematik und Mechanik 77(7) (1997), 519-526.

[32] F. dell'Isola and C. Woźniak, On phase transition layers in certain micro-damaged two-phase solids, International Journal of Fracture 83(2) (1997), 175-189.

[33] F. dell'Isola, L. Rosa and C. Woźniak, Dynamics of solids with micro periodic nonconnected fluid inclusions, Archive of Applied Mechanics 67(4) (1997), 215-228.

[34] F. dell'Isola, M. Guarascio and K. Hutter, A variational approach for the deformation of a saturated porous solid. A second gradient theory extending Terzaghi's effective stress principle, Arch. Appl. Mech. 70 (2000), 323-337.

[35] F. dell'Isola and K. Hutter, What are the dominant thermomechanical processes in the basal sediment layer of large ice sheets? Proceedings of the Royal Society A: Mathematical, Physical and Engineering Sciences 454(1972) (1998), 1169-1195.

[36] F. dell'Isola and S. Vidoli, Continuum modelling of piezoelectromechanical truss beams: An application to vibration damping, Arch. Appl. Mech. 68 (1998), 1-19.

[37] F. dell'Isola and S. Vidoli, Damping of bending waves in truss beams by electrical transmission lines with PZT actuators, Arch. Appl. Mech. 68 (1998), 626-636.

[38] F. dell'Isola, L. Rosa and C Woźniak, A micro-structured continuum modelling compacting fluid-saturated grounds: The effects of pore-size scale parameter, Acta Mechanica 127(1-4) (1998), 165-182.

[39] F. dell'Isola and A. Romano, A phenomenological approach to phase transition in classical field theory, International Journal of Engineering Science 25(11-12) (1987), 1469-1475.

[40] F. dell'Isola and A. Romano, On the derivation of thermomechanical balance equations for continuous systems with a nonmaterial interface, International Journal of Engineering Science 25(11-12) (1987), 14591468.

[41] A. DiCarlo and S. Quiligotti, Growth and balance. Mechanics Research Communications 29 (2002), 449456.

[42] M. Epstein and G.A. Maugin, Thermomechanics of volumetric growth in uniform bodies, International Journal of Plasticity 16 (2000), 951-978.

[43] V.A. Eremeyev and W. Pietraszkiewicz, Thermomechanics of shells undergoing phase transition, Journal of the Mechanics and Physics of Solids 59(7) (2011), 1395-1412.

[44] V.A. Eremeyev and W. Pietraszkiewicz, Phase transitions in thermoelastic and thermoviscoelastic shells, Archives of Mechanics 61(1) (2009), 41-67.

[45] V.A. Yeremeyev, A.B. Freidin and L.L. Sharipova, The stability of the equilibrium of two-phase elastic solids, Journal of applied mathematics and mechanics 71(1) (2007), 61-84. 
[46] V.A. Eremeyev and W. Pietraszkiewicz, The nonlinear theory of elastic shells with phase transitions, Journal of Elasticity 74(1) (2004), 67-86.

[47] V.A. Eremeev, A.B. Freidin and L.L. Sharipova, Nonuniqueness and stability in problems of equilibrium of elastic two-phase bodies, Doklady Physics 48(7) (2003), 359-363.

[48] J.D. Eshelby, The determination of the elastic field of an ellipsoidal inclusion and related problems, Proc $R$ Soc London A 241 (1957), 376-396.

[49] S. Federico, On the Linear Elasticity of Porous Materials, International Journal of Mechanical Sciences 52(2) (2010), 175-182, DOI:10.1016/j.ijmecsci.2009.09.006

[50] E. Garusi, A. Tralli and A. Cazzani, An unsymmetric stress formulation for reissner-mindlin plates: A simple and locking-free rectangular element, International Journal of Computational Engineering Science 5(3) (2004), 589-618.

[51] I.D. Ghiba, P. Neff, A. Madeo, L. Placidi, G. Rosi, The relaxed linear micromorphic continuum: existence, uniqueness and continuous dependence in dynamics. arXiv:1308.3762 submitted to Math. Mech. Solids.

[52] I. Giorgio, A. Culla and D. Del Vescovo, Multimode vibration control using several piezoelectric transducers shunted with a multiterminal network, Arch. Appl. Mech. 79 (2009), 859-879.

[53] M. A. Goodman and S. C. Cowin, A continuum theory for granular materials, Arch. Ration. Mech. An. 44 (1972), 249-266.

[54] A. Grillo, S. Federico, G. Wittum, Growth, mass transfer, and remodeling in fiber-reinforced, multiconstituent materials, International Journal of Non-Linear Mechanics 47 (2012), 388-401.

[55] M. V. Hillsley and J.A. Frangos, Review: Bone tissue engineering: The role of interstitial fluid flow, Biotechnology and Bioengineering 43(7) (1994), 573-581.

[56] R. Huiskes, H. Weinans, H. J. Grootenboer, M. Dalstra, B. Fudala and T. J. Slooff, Adaptive boneremodeling theory applied to prosthetic-design analysis, J. Biomech. 20 (1987), 1135-50.

[57] J. Klein-Nulend, P. J. Nijweide, E. H. Burger, Osteocyte and bone structure, Current Osteoporosis Reports 1 (2003), 5-10.

[58] I.M. Koç, A. Carcaterra, Z. Xu, A. Akay, Energy sinks: Vibration absorption by an optimal set of undamped oscillators, Journal of the Acoustical Society of America 118(5) (2005), 3031-3042.

[59] T. Lekszycki, Modelling of bone adaptation based on an optimal response hypothesis, Meccanica 37 (2002), 343-354.

[60] T. Lekszycki and F. dell'Isola, A mixture model with evolving mass densities for describing synthesis and resorption phenomena in bones reconstructed with bio-resorbable materials, J. Applied Math and Mech. (ZAMM) 92 (2012), 426-444.

[61] A. Luongo, A. Di Egidio and A. Paolone, Multiscale analysis of defective multiple-Hopf bifurcations, Computers and Structures 82(31-32) (2004), 2705-2722.

[62] A. Luongo, A. Paolone and A. Di Egidio, Multiple Timescales Analysis for 1:2 and 1:3 Resonant Hopf Bifurcations, Nonlinear Dynamics 34(3-4) (2003), 269-291.

[63] A. Luongo and A. Paolone, On the reconstruction problem in the multiple time-scale method, Nonlinear Dynamics 19(2) (1999), 133-156.

[64] A. Luongo and A. Paolone, Perturbation Methods for Bifurcation Analysis from Multiple Nonresonant Complex Eigenvalues, Nonlinear Dynamics 14(3) (1997), 193-210.

[65] A. Luongo, Perturbation methods for nonlinear autonomous discrete-time dynamical systems, Nonlinear Dynamics 10(4) (1996), 317-331.

[66] A. Madeo, P. Neff, I.D. Ghiba, L. Placidi and G. Rosi, Wave propagation in relaxed micromorphic continua: modeling metamaterials with frequency band-gaps, Continuum Mechanics and Thermodynamics DOI: 10.1007/s00161-013-0329-2:1-20, (2013).

[67] A. Madeo, F dell'Isola, F. Darve, A continuum model for deformable, second gradient porous media partially saturated with compressible fluids, Journal of the Mechanics and Physics of Solids 61(11) (2013), 2196-2211.

[68] A. Madeo, I. Djeran-Maigre, G. Rosi and C. Silvani, The effect of fluid streams in porous media on acoustic compression wave propagation, transmission, and reflection, Continuum Mech. Therm. (2012). 
[69] A. Madeo, D. George, T. Lekszycki, M. Nierenberger and Y. Rémond, A second gradient continuum model accounting for some effects of micro-structure on reconstructed bone remodeling, Comptes Rendus Mécanique 340 (2012) 575-589.

[70] A. Madeo, T. Lekszycki and F. dell'Isola, A continuum model for the bio-mechanical interactions between living tissue and bio-resorbable graft after bone reconstructive surgery, Comptes Rendus Mécanique 339 (2011), 625-640.

[71] A. Madeo, F. dell'Isola, N. Ianiro and G. Sciarra, A variational deduction of second gradient poroelasticity II: An application to the consolidation problem, Journal of Mechanics of Materials and Structures 3(4) (2008), 607-625.

[72] R. B. Martin, Porosity and specific surface of bone, CRC Critical reviews in Biomedical Engineering 10 Boca Raton FL, CRC Press (1984), 179-222.

[73] C. Maurini, F. dell'Isola and D. Del Vescovo, Comparison of piezoelectronic networks acting as distributed vibration absorbers, Mech. Syst. Signal Pr. 18 (2004), 1243-1271.

[74] C. Maurini, J. Pouget and F. dell'Isola, On a model of layered piezoelectric beams including transverse stress effect, Int. J. Solids Struct. 41 (2004), 4473-4502.

[75] C. Maurini, J. Pouget and F. dell'Isola, Extension of the Euler-Bernoulli model of piezoelectric laminates to include 3D effects via a mixed approach, Computers and Structures 84 (2006), 1438-1458.

[76] P. Neff, I.D. Ghiba, A. Madeo, L. Placidi and G. Rosi, A unifying perspective: the relaxed linear micromorphic continuum, Continuum Mechanics and Thermodynamics DOI: 10.1007/s00161-013-0322-9, (2013).

[77] P. Neff and S. Forest, A geometrically exact micromorphic model for elastic metallic foams accounting for affine microstructure. Modelling, existence of minimizers, identification of moduli and computational results, J. Elasticity 87 (2007), 239-276.

[78] P. Neff, The Cosserat couple modulus for continuous solids is zero viz the linearized Cauchy-stress tensor is symmetric, Z. Angew. Math. Mech. 86 (2006) 892-912.

[79] G. Oliveto and M. Cuomo, Incremental analysis of plane frames with geometric and material nonlinearities, Engineering Structures 10(1) (1988), 2-12.

[80] H. Petite, V. Viateau, W. Bensaïd, A. Meunier, C. de Pollak, M. Bourguignon, K. Oudina, L. Sedel and G. Guillemin, Tissue-engineered bone regeneration, Nat Biotechnol. 18 (2000), 959-963.

[81] W. Pietraszkiewicz, V. Eremeyev, V. Konopińska, Extended non-linear relations of elastic shells undergoing phase transitions, ZAMM Zeitschrift für Angewandte Mathematik und Mechanik 87(2) (2007), 150-159.

[82] L. Placidi, F. dell'Isola, N. Ianiro and G. Sciarra, Variational formulation of pre-stressed solid-fluid mixture theory, with an application to wave phenomena, European Journal of Mechanics - A/Solids 27 (2008), 582606.

[83] S. Quiligotti, G. A. Maugin and F. dell'Isola, An eshelbian approach to the nonlinear mechanics of constrained solid-fluid mixtures, Acta Mechanica 160 (2003), 45-60.

[84] Y. Ramaswamy, D. R. Haynes, G. Berger, R. Gildenhaar and H. Lucas, Bioceramics composition modulate resorption of human osteoclasts, Journal of Materials Science: Materials in Medicine 16 (2005), 1199-1205.

[85] E. Reccia, A. Cazzani and A. Cecchi, FEM-DEM Modeling for Out-of-plane Loaded Masonry Panels: A Limit Analysis Approach, Open Civil Engineering Journal 6(1) (2012), 231-238.

[86] A. Rinaldi and L. Placidi, A microscale second gradient approximation of the damage parameter of quasibrittle heterogeneous lattices, (2013) DOI: 10.1002/zamm.201300028.

[87] A. Rinaldi, A rational model for 2D Disordered Lattices Under Uniaxial Loading, Int. J. Damage Mech. 18 (2009), 233-257.

[88] A. Rinaldi and Y.-C. Lai, Statistical damage theory of 2 d lattices: energetics and physical foundations of damage parameter, Int. J. Plasticity 23 (2007), 1796-1825.

[89] G. Rosi, R. Paccapeli, F. Ollivier and J. Pouget, Optimization of piezoelectric patches positioning for passive sound radiation control of plates, J. Vib. Control (2012).

[90] G. Rosi, J. Pouget and F. dell'Isola, Control of sound radiation and transmission by a piezoelectric plate with an optimized resistive electrode, Eur. J. Mech. A-Solids 29 (2010), 859-870.

[91] C. T. Rubin and L.E. Lanyon, Osteoregulatory nature of mechanical stimuli: Function as a determinant for adaptive remodeling in bone, J. Orthopaed. Res. 5 (1987), 300-310. 
[92] J. A. Sanz-Herrera, J. M. García-Aznar and M. Doblaré, Micro-macro numerical modelling of bone regeneration in tissue engineering, Computer methods in Applied Mechanics and Engineering 197 (2008), 3092-3107.

[93] J. A. Sanz-Herrera and A. R. Boccaccini, Modelling bioactivity and degradation of bioactive glass based tissue engineering scaffolds, International Journal of Solids and Structures 48 (2010), 257-268.

[94] A. F. Schilling, W. Linhart, S. Filke, M. Gebauer, T. Schinke, J. M. Rueger and M. Amling, Resorbability of bone substitute biomaterials by human osteoclasts, Biomaterials 25 (2004), 3963-3972.

[95] G. Sciarra, F. dell'Isola and K. Hutter, A solid-fluid mixture model allowing for solid dilatation under external pressure, Continuum Mech. Therm. 13 (2001), 287-306.

[96] S. Sethuraman, L. S. Nair, S. El-Amin, M. T. Nguyen, A. Singh, N. Krogman, Y. E. Greish, H. R. Allcock, P. W. Brown and C. T. Laurencin, Mechanical properties and osteocompatibility of novel biodegradable alanine based polyphosphazenes: Side group effects, Acta Biomater. 6 (2010), 1931-1937.

[97] A. Tricoteaux, E. Rguiti, D. Chicot, L. Boilet, M. Descamps, A. Leriche and J. Lesage, Influence of porosity on the mechanical properties of microporous $\beta$-TCP bioceramics by usual and instrumented Vickers microindentation, Journal of the European Ceramic Society 31 (2011), 1361-1369.

[98] A. H. Yusop, A. A. Bakir, N. A. Shaharom, M. R. Abdul Kadir and H. Hermawan, Porous Biodegradable Metals for Hard Tissue Scaffolds: A Review, Int J Biomater. (2012), 1-10, Article ID 641430.

[99] M. Zanetta, N. Quirici, F. Demarosi, M. C. Tanzi, L. Rimondini and S. Farè, Ability of polyurethane foams to support cell proliferation and the differentiation of MSCs into osteoblasts, Acta Biomater. 5 (2009), 1126-1136.

[100] H. Zhou, J. G. Lawrence and S. B. Bhaduri, Fabrication aspects of PLA-CaP/PLGA-CaP composites for orthopedic applications: a review, Acta Biomater. 8 (2012), 1999-2016.

Ugo Andreaus

Dipartimento di Ingegneria Strutturale e Geotecnica

Facoltà di Ingegneria Civile e Industriale

Università Sapienza di Roma

Via Eudossiana 18, 00184 - Roma, Italia

e-mail: ugo.andreaus@uniroma1.it

Ivan Giorgio

Dipartimento di Ingegneria Strutturale e Geotecnica

Facoltà di Ingegneria Civile e Industriale

Università Sapienza di Roma

Via Eudossiana 18, 00184 - Roma, Italia

International Research Center for the Mathematics and Mechanics of Complex Systems 'M\&MoCS',

Università de L'Aquila, Italy

e-mail: ivan.giorgio@uniroma1.it

Angela Madeo

Université de Lyon

INSA (Institut National des Sciences Appliquées)

20, avenue Albert-Einstein, 69621 Villeurbanne cedex, France

International Research Center for the Mathematics and Mechanics of Complex Systems 'M\&MoCS',

Università de L'Aquila, Italy

e-mail: angela.madeo@insa-lyon.fr 